\title{
dad: an R Package for Visualisation, Classification and Discrimination of Multivariate Groups Modelled by their Densities
}

\author{
by Rachid Boumaza, Pierre Santagostini, Smail Yousfi and Sabine Demotes-Mainard
}

\begin{abstract}
Multidimensional scaling (MDS), hierarchical cluster analysis (HCA), and discriminant analysis (DA) are classical techniques which deal with data made of $n$ individuals and $p$ variables. When the individuals are divided into $T$ groups, the $\mathrm{R}$ package dad associates with each group a multivariate probability density function and then carries out these techniques on the densities, which are estimated by the data under consideration. These techniques are based on distance measures between densities: chi-square, Hellinger, Jeffreys, Jensen-Shannon, and $L^{p}$ for discrete densities, Hellinger, Jeffreys, $L^{2}$, and 2-Wasserstein for Gaussian densities, and $L^{2}$ for numeric non-Gaussian densities estimated by the Gaussian kernel method. Practical methods help the user to give meaning to the outputs in the context of MDS and HCA and to look for an optimal prediction in the context of DA based on the one-leave-out misclassification ratio. Some functions for data management or basic statistics calculations on groups are annexed.
\end{abstract}

\section{Introduction}

Techniques such as multidimensional scaling, hierarchical cluster analysis, and discriminant analysis are often used for multivariate data analysis to visualize, organize observations into groups, and model class structure, respectively. These techniques deal with data of the "individuals $\times$ variables" type (Mardia et al., 1979; Krzanowski, 1988), commonly stored in objects of class data frame. These techniques are available in the R packages stats, MASS (Venables and Ripley, 2002), ade4 (Dray et al., 2007), FactoMineR (Lê et al., 2008), cluster (Maechler et al., 2019).

In the case where the individuals are organized into occasions or groups, the analyst could be interested in taking into account this data organization by associating with each occasion a mathematical object and performing multivariate techniques on these objects. In the dad package (Boumaza et al., 2021), devoted to such data, the objects are probability density functions. These densities are either all continuous (numeric data with Lebesgue measure as reference measure) or all discrete (categorical data with counting measure as reference measure) and are subjected to the following analyses:

- Multidimensional scaling (MDS) of probability density functions aims to visualize a set of densities (or occasions) so that the distances between the densities are preserved as well as possible;

- Hierarchical cluster analysis (HCA) of probability density functions is used to divide a set of densities (or occasions) into clusters so that the densities of the same cluster are as similar as possible and are dissimilar from those of the other clusters;

- Discriminant analysis (DA) of probability density functions deals with the same kind of data, knowing a partition of the densities (or occasions) into classes. Its first objective is to learn how the a priori classes can be explained by the distances between these densities. Then, if the training step is judged satisfactory according to a criterion named misclassification ratio, its second objective is to classify a new density whose class is unknown (Boumaza, 2004).

These three multivariate techniques constitute the core of this work. Theoretically, the dad package handles probability density functions and considers multi-group data as samples allowing their estimation. The densities could be considered as functional data processed by packages like fda (Ramsay et al., 2020), fda.usc (Febrero-Bande and de la Fuente, 2012), or fdadensity (Petersen et al., 2019), or as compositional data processed by packages like compositions (Tsagris and Athineou, 2020), Compositional (van den Boogaart et al., 2020), or robCompositions (Filzmoser et al., 2018). The differences or similarities with these approaches are the subjects of part of the discussion of the manuscript (Discussion and concluding remarks Section).

These three multivariate techniques are essentially based on distance indices between probability density functions. Literature abounds with such indices: as an example, the encyclopedia of distances of Deza and Deza (2013, p. 235-245) lists some forty. The dad package proposes to calculate ten of them among the most common by considering the case of discrete densities and that of continuous densities. 
The results returned by the three previous multivariate techniques depend on the distance index used. This is illustrated by simple examples in the context of HCA (Appendix A) or DA (Appendix B), and criteria of distance choice are proposed in the Practical advice Section.

Thus, for each distance index, the dad package implements:

- its calculation for two densities whose type and parameters are known,

- its estimation for two densities for which there are two samples which allow the estimation of their parameters,

- the generalization of each previous calculation for $T(T>2)$ densities taken two by two, the result of which is a symmetric matrix.

In order to avoid unnecessary redundancies in the entry of data characterizing the groups as these characteristics are the same for all the individuals of the same group, we considered it useful to organize the data in a list of data frames (object of class folderh). Also, in order to calculate some statistics (means, covariance matrices...) per group or distances between each pair of groups, we considered it useful to store the individuals of each group in one data frame and these data frames in a list (object of class folder). Appendix $C$ details the rationale for introducing these object classes. The functions of the package dad implementing the three main techniques (MDS, HCA, DA) apply to such objects. The functions of data management or elementary calculation (means, variance or correlation matrices, moments) applying to these objects could have been made invisible in the package without affecting the presentation of the main techniques. However, to facilitate the work of the analyst interested in processing multi-group data or in experimenting with other multivariate techniques on such data, we have kept them visible. Some of them are presented in Appendix C and Appendix D.

So, the presentation of the dad package will begin with a description of the data considered in the previous techniques (Multi-group data: examples and organization Section). We will then present functions for the calculation of the distance or divergence measures between discrete densities and between Gaussian densities. The special case of non-Gaussian densities estimated with the Gaussian kernel method is also considered (Distance / divergence between densities Section). Then, we will present the functions implementing the three techniques introduced above for the processing of multi-group data: multidimensional scaling (MDS of densities Section), hierarchical cluster analysis (HCA of densities Section), and discriminant analysis (DA of densities Section). Finally, we will give some practical advice (Practical advice Section) and briefly highlight some similarities with functions of other $\mathrm{R}$ packages (Discussion and concluding remarks Section). A summary and appendices complete this presentation.

\section{Multi-group data: examples and organization}

For MDS and HCA, the data $\mathbf{X}$ (Table 1a) of interest have three kinds of objects: occasions $\times$ individuals $\times$ variables. The occasions define a partition of the individuals on which the variables are measured. If $T$ denotes the number of occasions, for each $t$ in $\{1, \ldots, T\}$, the rows of the table $\mathbf{X}_{t}$ correspond to $n_{t}$ observations $\mathbf{x}_{t 1}, \ldots, \mathbf{x}_{t n_{t}}$ of $X_{t}$ a random vector with $p$ components.

For DA, the data of interest are similar to the previous ones with the difference that we have two categories of occasions. The first category consisting of $T$ occasions is partitioned into $K$ subsets deriving from a factor $G$ defined on occasions (Table 2). The second category consists of occasions, numbered $T+1, \ldots$ for which we have data of type $\mathbf{X}$ but not the value of $G$.

\section{Datasets}

The data of the following examples are available in the dad package as lists of data frames or arrays, and are loaded by means of the usual data function.

1. Archaeological data: the data are stored in castles. dated, a list of two data frames whose description is detailed in the subsection Introductory example of Appendix C. The data frame castles. dated $\$$ stones in which for each of $T=68$ Alsatian castles (occasions), $p=4$ numerical characteristics are measured on a batch of stones (individuals) used to build the castle. The objective is to:

- visualize the castles by points in a space of reduced dimension so that the castles having stones of similar dimensions are close to each other and those having stones of very different dimensions are distant;

- highlight which characteristics of the stones are at the origin of these small or large distances. 


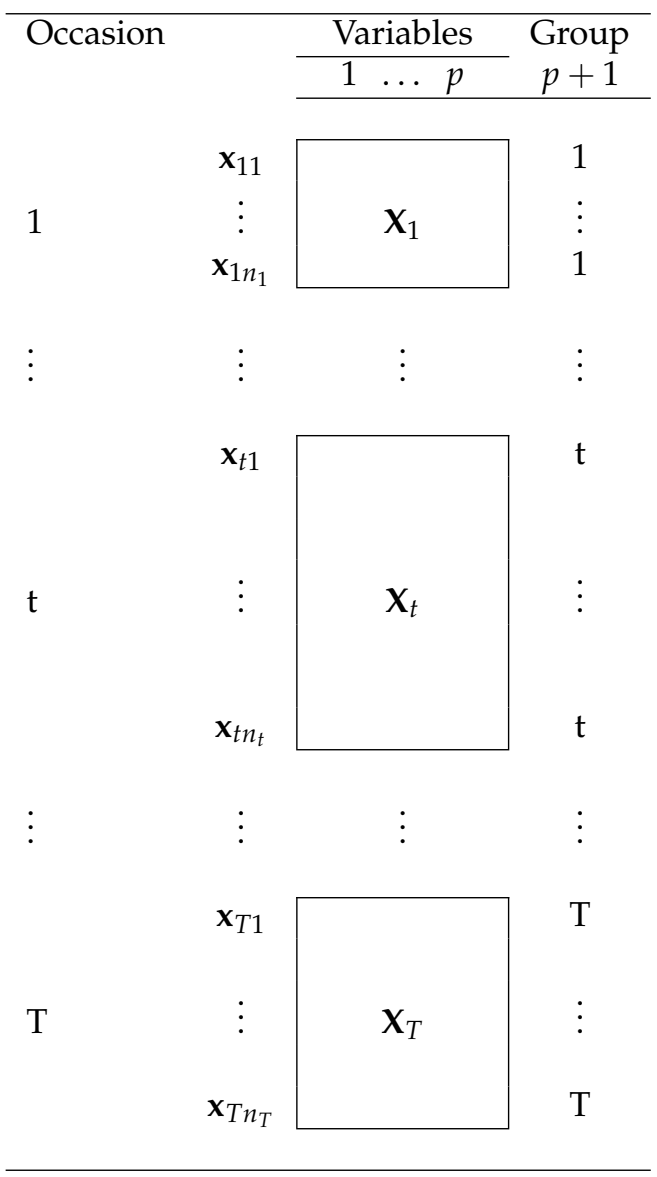

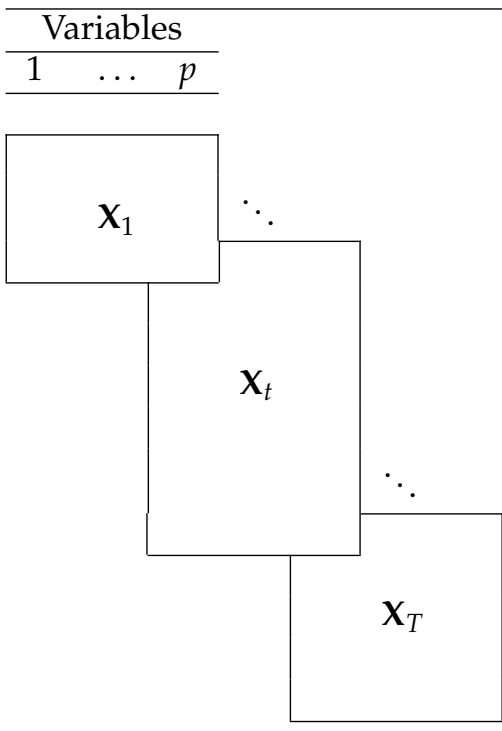

(b) Data folder

(a) Data frame

Table 1: For each occasion $t=1, \ldots, T$, the same $p$ variables are observed for $n_{t}$ individuals. The data frame (a) consists of $(p+1)$ columns, the last one is a factor designating the occasion. The data folder (b) consists of $T$ data frames each one having the same $p$ column names.

The 68 castles are dated from the period 1140-1650, which is divided into six intervals, numbered 1 to 6 , separated by the following cutoff points: $1175,1210,1245,1280,1350$. The building periods are available in the data frame castles. dated\$periods. The first objective is to analyse the relations between these periods and the previous visualization of the castles. The second objective is to predict the building period of 67 non-dated castles the data of which are in the data frame castles. nondated\$stones.

This archaeological example (Rudrauf and Boumaza, 2001) was at the origin of the multi-group techniques presented in this work. It illustrates well the MDS and DA techniques even if, as we will see when processing the data, from the archeology point of view, the results are not very satisfactory and have only an indicative value.

2. Agronomic data: the data are stored in the folderh object varietyleaves consisting of two data frames variety and leaves, and a key rose that is the column name common to the two data frames which connects them. The first data frame varietyleaves $\$$ leaves is made up of 581 rows (leaves of $T=31$ rosebushes) and 5 columns corresponding to the number of the rosebush to which the leaf belongs and $p=4$ numerical characteristics: number of its leaflets, length of its rachis, then the length, and width of its main leaflet. The second data frame varietyleaves $\$$ variety is made up of $T$ rows and two columns: the number of the rosebush and its variety. There are $K=6$ varieties (Table 3 ). This example will illustrate the DA technique. The objective of which is to predict the variety of one plant from measures of several leaves of the plant.

3. Sensory data: the data frame roses in which each of $T=10$ photographs of rosebushes (occasions) was evaluated 3 times, by 14 assessors, for $p=16$ numerical characteristics, giving a table with 16 columns and 42 rows per rosebush, for a total of 420 rows. In this case, an individual is a couple (assessor, evaluation session). The objective is to visualize the roses and then create clusters of roses that are as similar as possible. A part of these data will illustrate the 


\begin{tabular}{|c|c|}
\hline Occasion & $G$ \\
\hline 1 & 1 \\
\hline$\vdots$ & $\vdots$ \\
\hline$T_{1}$ & 1 \\
\hline$T_{1}+1$ & 2 \\
\hline$\vdots$ & $\vdots$ \\
\hline$T_{1}+T_{2}$ & 2 \\
\hline \multicolumn{2}{|l|}{$\vdots$} \\
\hline$T_{1}+\ldots+T_{K-1}+1$ & $\bar{K}$ \\
\hline$\vdots$ & $\vdots$ \\
\hline$T=T_{1}+\ldots+T_{K-1}+T_{K}$ & K \\
\hline$T+1$ & Not available \\
\hline$\vdots$ & $\vdots$ \\
\hline
\end{tabular}

Table 2: Each occasion $t(t=1, \ldots, T)$ matches a table with $n_{t}$ rows and $p$ columns (see Table 1$)$. The variable $G$ defined on the occasions takes values $\{1, \ldots, K\}$. For each $k=1, \ldots, K$, the value $k$ is taken $T_{k}$ times. The $G$ values of the occasions $T+1, \ldots$ are not available and have to be predicted.

techniques MDS and HCA.

4. Surveys - census data over years - : from each census conducted in France (INSEE, 2018) during $T=7$ different years $(1968,1975,1982,1990,2010$, and 2015), we extract the population of active individuals aged 25 to 54 and $p=2$ categorical variables: diploma (4 levels) and socio-professional group (6 levels). From the initial data collected by INSEE, we build a list of $T=7$ arrays of dimension $(4,6)$, which is stored in dspg object. The objective is to visualize the years and to highlight, when they exist, the temporal evolutions of the frequencies. The MDS technique seems suitable to achieve this objective.

5. Surveys - census data 2015 by department - : from the census of the year 2015 (INSEE, 2018), we consider the same kind of data and organize them in a list of $T=96$ arrays, which correspond to the 96 departments of metropolitan France. This list is available in the dspgd2015 object. As for the sensory data, the objective is to visualize the departments and then create clusters of departments that are as similar as possible. In order to give meaning to the clusters, a common and advisable practice is to couple the techniques HCA and MDS.

In the last two examples, the densities are discrete and are given only for illustration. Their detailed presentation is in the dad vignette mds-discrete-distributions.

\section{Data management}

The previously collected data can be organized into:

- A single data frame (Table $1 a$ ), by vertically concatenating the $T$ tables $\mathbf{X}_{t}$, with $p$ columns and appending a factor column designating the occasion, or

- A list of $T$ data frames (Table 1b), each having $p$ columns, as an object from an S3 class named folder (see Appendix C).

\begin{tabular}{|l|c|c|l|}
\hline Variety & $\begin{array}{c}\text { Number } \\
\text { of plants }\end{array}$ & $\begin{array}{c}\text { Number } \\
\text { of leaves }\end{array}$ & $\begin{array}{l}\text { Number of leaves } \\
\text { per plant }\end{array}$ \\
\hline Canary & 6 & 65 & $=8+14+11+16+8+8$ \\
Electron & 3 & 42 & $=9+8+25$ \\
Lili Marleen & 6 & 65 & $=15+10+5+7+15+13$ \\
Pussta & 6 & 200 & $=35+47+29+20+37+32$ \\
Starina & 5 & 105 & $=18+17+19+33+18$ \\
White Meillandina & 5 & 104 & $=23+19+16+22+24$ \\
\hline
\end{tabular}

Table 3: Numbers of plants and leaves per variety and number of leaves per plant. 
To carry out discriminant analysis, the training step requires an a priori division of the occasions into clusters, that is, a factor $G$ with $K$ levels defined from the occasion set (Table 2). The predicting step is to assign a level for each occasion whose value of the factor $G$ is not available. The data, therefore, consist of two data frames linked by a hierarchical relationship " 1 to $N$ ". Each row of the data frame in Table 2 is, thus, matched to several rows of the data frame in Table 1a. The list of these two data frames is an object of S3 class folderh (hierarchical folder) built by the function folderh (see Appendix C).

Notice that in the presentation of the data tables, we arranged them so that the individuals (rows of Table 1a) of the same occasion are neighbors and so that the occasions (rows of Table 2) of the same class are neighbors. However, in the dad package, such a layout is obviously not necessary. Only the factors Group (Table 1a) and $G$ (Table 2) must be given.

\section{Distance/divergence between densities}

In the first subsection, we present the indices which operate on discrete densities. In the second subsection, we consider the indices which operate on Gaussian densities and have an analytical expression depending on the parameters of the densities: means, variances, and covariances. Therefore, these indices can easily be estimated from the parameter estimates. In the third subsection, we present an index based on the estimation of densities on $\mathbb{R}^{p}$ by the Gaussian kernel method. This index offers the advantage of being easily calculable even for non-Gaussian continuous densities.

The main techniques of the dad package depend on the distance index used (Appendix A and B). A brief practical guidance on the choice of distance index is provided in Practical advice Section.

\section{Calculation of distances/divergences between discrete densities}

\begin{tabular}{ll}
\hline Name & Expression \\
\hline Symmetric chi-square & $\sum_{x}\left(p_{1}(x)-p_{2}(x)\right)^{2} /\left(p_{1}(x)+p_{2}(x)\right)$ \\
Hellinger & $\left(2 \sum_{x}\left(\sqrt{p_{1}(x)}-\sqrt{p_{2}(x)}\right)^{2}\right)^{\frac{1}{2}}$ \\
Jeffreys & $\sum_{x}\left(p_{1}(x)-p_{2}(x)\right) \ln \left(p_{1}(x) / p_{2}(x)\right)$ \\
Jensen-Shannon & $\sum_{x}\left(p_{1}(x) \ln \left(2 p_{1}(x) /\left(p_{1}(x)+p_{2}(x)\right)\right)\right.$ \\
& $\left(\sum_{x}\left|p_{1}(x)-p_{2}(x)\right|^{p}\right)^{\frac{1}{p}}$ \\
&
\end{tabular}

Table 4: Distance indices between two discrete densities $p_{1}$ and $p_{2}$ on the same finite support the states of which are denoted $x$ in the formulas. The sums of the formulas are taken over all the states of the support (Deza and Deza, 2013). The corresponding dad functions are: ddchisqsympar, ddhellingerpar, ddjeffreyspar, ddjensenpar, and ddlppar.

Table 4 lists the expressions of distance indices of two discrete densities and the dad functions associated with them. The set of the states of these densities can be either the set of the levels of one categorical variable or the Cartesian product of the $q$ sets of the levels of $q$ categorical variables as in the following example with $q=2$.

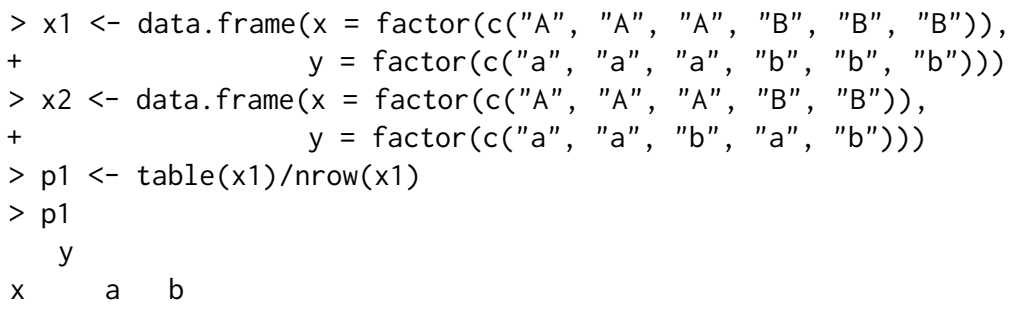




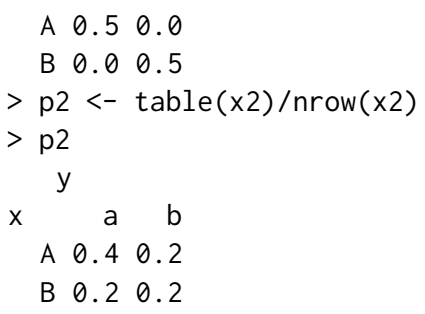

The $L 1$ distance and Jeffreys divergence between the densities $p 1$ and $p 2$ are computed as follows.

$>\operatorname{ddlppar}(\mathrm{p} 1, \mathrm{p} 2)$

[1] 0.8

$>$ ddjeffreyspar (p1, p2)

[1] Inf

The Jensen-Shannon index is equal to the entropy or average quantity of information of the distribution $\left(p_{1}+p_{2}\right) / 2$, from which we subtract the sum of the entropies of $p_{1}$ and $p_{2}$. The other indices are based on the sum of the differences, possibly weighted, between $p_{1}$ and $p_{2}$ in each state $x$, unlike the Jensen-Shannon index, which is somewhat more global. These indices are compared in the subsection Simulated discrete data of Appendix B.

\section{Calculation of distances/divergences between Gaussian densities}

Table 5 lists the parametric expressions of distance indices of multivariate densities and the dad functions associated with them. For the univariate case, the expressions are easily deduced.

\begin{tabular}{ll}
\hline Name & Expression \\
\hline Hellinger ${ }^{\text {(a) }}$ & $\left(2-2^{\frac{p}{2}+1} \operatorname{det}(\Sigma V)^{\frac{1}{4}} \operatorname{det}(\Sigma+V)^{-\frac{1}{2}} \exp \left(-\frac{1}{4}\|\mu-m\|_{(\Sigma+V)^{-1}}^{2}\right)\right)^{\frac{1}{2}}$ \\
Jeffreys $^{(\mathrm{b})}$ & $2^{-1}\|\mu-m\|_{\Sigma^{-1}+V^{-1}}^{2}+2^{-1} \operatorname{tr}\left((\Sigma-V)\left(V^{-1}-\Sigma^{-1}\right)\right)$ \\
L2 $^{\text {(c) }}$ & $\left((2 \pi)^{-\frac{p}{2}} \operatorname{det}(2 \Sigma)^{-\frac{1}{2}}+(2 \pi)^{-\frac{p}{2}} \operatorname{det}(2 V)^{-\frac{1}{2}}\right.$ \\
& $\left.\left(2-2(2 \pi)^{-\frac{p}{2}} \operatorname{det}(\Sigma+V)^{-\frac{1}{2}} \exp \left(-\frac{1}{2}\|\mu-m\|_{(\Sigma+V)^{-1}}^{2}\right)\right)^{\frac{1}{2}} \operatorname{det}(\Sigma V)^{\frac{1}{4}} \operatorname{det}(\Sigma+V)^{-\frac{1}{2}} \exp \left(-\frac{1}{2}\|\mu-m\|_{(\Sigma+V)^{-1}}^{2}\right)\right)^{\frac{1}{2}}$ \\
L2N $^{(\mathrm{d})}$ & $\left(\|\mu-m\|_{I_{p}}^{2}+\operatorname{tr}\left(\Sigma+V-2\left(V^{\frac{1}{2}} \Sigma V^{\frac{1}{2}}\right)^{\frac{1}{2}}\right)\right)^{\frac{1}{2}}$
\end{tabular}

Table 5: Distance indices between the multivariate Gaussian densities $f \equiv N(\mu, \Sigma)$ and $g \equiv N(m, V)$. (a) The corresponding dad function is hellingerpar. It is the $L^{2}$ distance between the square roots of the densities $f$ and $g$. (b) The Jeffreys divergence is the symmetrized Kullback-Leibler divergence. Its corresponding function is jeffreyspar. (c) The corresponding function of the $L^{2}$ distance is dist12dpar. (d) L2N, named also normalized $L^{2}$ distance, stands for the $L^{2}$ distance between $f /\|f\|_{L^{2}}$ and $g /\|g\|_{L^{2}}$ and its value is almost similar to the Hellinger distance. Its corresponding function is distl2dnormpar. (e) $I_{p}$ stands for the identity matrix of order $p$. The corresponding function is wassersteinpar.

For example, the Jeffreys divergence is respectively carried out with the jeffreyspar or jeffreys functions depending on whether the calculations are respectively based on parameters or samples.

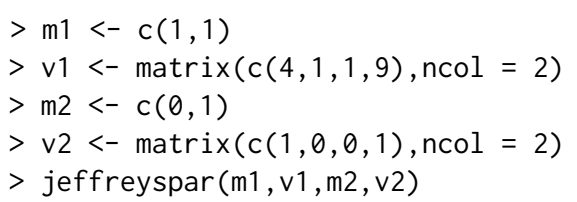


[1] 5.314286

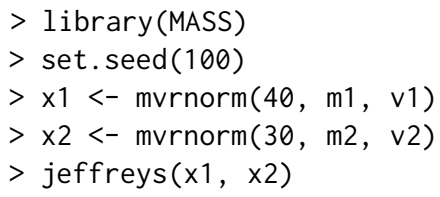

[1] 6.780999

All these indices are based on a combination of the difference between the means $\mu$ and $m$ and the dissimilarity between the covariance matrices $\Sigma$ and $V$. In the case of equal means, the distance index between Gaussian densities reduces to a dissimilarity between covariance matrices. If the covariance matrices are equal, all these indices reduce to an index of the distance between these means, this index being dependent on the common variance matrix, with the exception of the Wasserstein index, which uses the identity matrix. These indices are also compared in the subsection Simulated Gaussian data of Appendix B.

\section{Calculation of $L^{2}$ distances between continuous non-Gaussian densities estimated by the kernel method}

Except for the $L^{2}$ distances, the extension of the other distance indices of Table 5 to any estimated densities still requires a lot of work. Indeed, we experimented with calculating distance indices using numerical integration methods but computation times were so long in the multidimensional case that we did not implement them in the dad package. The solution we recommend is to estimate the densities by the Gaussian kernel method and use the $L^{2}$ distances.

- Density estimation with the Gaussian kernel method. The probability densities $f_{t}$ are estimated by the Gaussian kernel method:

$$
\hat{f}_{t}(\mathbf{z})=\frac{1}{n_{t}\left|\mathbf{h}_{t}\right|^{1 / 2}} \frac{1}{(2 \pi)^{p / 2}} \sum_{i=1}^{n_{t}} \exp \left(-\frac{1}{2}\left(\mathbf{z}-\mathbf{x}_{t i}\right)^{\top} \mathbf{h}_{t}^{-1}\left(\mathbf{z}-\mathbf{x}_{t i}\right)\right),
$$

where $\mathbf{h}_{t}$ is the non-singular bandwidth matrix and $\left|\mathbf{h}_{t}\right|$ its determinant. This matrix may be provided by the user, or calculated directly according to the AMISE criterion, with reference to the normal distribution (Wand and Jones, 1995), that is:

$$
\mathbf{h}_{t}=h_{t} \widehat{\mathbf{V}}_{t}^{1 / 2},
$$

with:

$$
h_{t}=\left(\frac{4}{n_{t}(p+2)}\right)^{\frac{1}{p+4}}
$$

- Calculation of $L^{2}$ distances between estimated non-Gaussian densities. The calculation of the inner product is carried out with the $12 \mathrm{~d}$ function using a sample per density: $\mathrm{x} 1$ and $\mathrm{x} 2$; the result derives from the estimation (1) of the densities, the bilinearity of the inner product, and a formula of integral calculus (Wand and Jones, 1995, p. 101). Then, the $L^{2}$-distance is directly deduced. This calculation is carried out with the distl2d function.

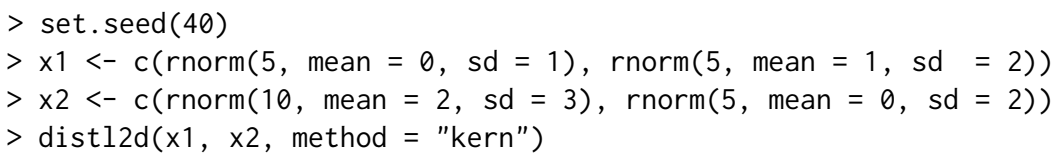

[1] 0.2562896

The normalized $L^{2}$ distance between densities is also possible. Although it has the disadvantage of being time-consuming, it has some similarities to Hellinger's distance, and in the Gaussian case, the two distances have almost the same expression (Table 5).

\section{MDS of densities}

Since the function of dad package implementing multidimensional scaling of probability density functions is a direct application of the function cmdscale of $R$, it is briefly recalled in the case of 
continuous densities. The mathematical aspects of this method have been dealt with in several works (Delicado (2011) as MDS; Boumaza (1998), Kneip and Utikal (2001), and Yousfi et al. (2015) as functional PCA). In this section, we will privilege the presentation in MDS form, which offers greater flexibility in the choice of the distance between densities, while taking inspiration from the method of interpretation of the results of PCA developed in Boumaza et al. (2015) in order to interpret the scores resulting from MDS.

\section{Brief presentation of the method}

Given $T$ densities and $\left(\delta_{t s}\right)_{1 \leq t, s \leq T}$ the distances/divergences between each pair of them, the MDS technique looks for a representation of the densities by $T$ points in a low dimensional space such that the distances between these points are as similar as possible to the $\left(\delta_{t s}\right)$ (Cox and Cox, 2001). In $\mathrm{R}$, this multidimensional positioning technique is performed by the cmdscale function, whose main argument is the symmetric matrix of distances and the main output is the matrix of coordinates.

If the densities are assumed to be Gaussian, we can use the Hellinger distance, the Jeffreys divergence, the 2-Wasserstein distance, or the $L^{2}$ distance (Table 5). If they are not expected to be Gaussian, they are estimated using the Gaussian kernel method, and the only available distance for the moment is the $L^{2}$-distance (Calculation of $L^{2}$ distances between continuous non-Gaussian densities estimated by the kernel method Section).

The dad package includes functions for all the calculations required to implement such a method and to interpret its outputs:

- The fmdsd function which performs multidimensional scaling and generates scores;

- The plot function which generates graphics representing the densities on the factorial axes;

- The interpret function which returns other aids to interpretation based on the moments of the variables.

\section{The fmdsd function}

MDS of densities can be carried using the fmdsd function, which applies to an object of the class folder (Table 1b). The future or to a data frame and a grouping variable (Table 1a). It is built on the cmdscale function of R. In addition to the add argument of cmdscale, the fmdsd function has three sets of optional arguments. The first, consisting of gaussiand, windowh, and distance, controls the method used to estimate the densities and their distances (Distance/divergence between densities Section). The second consists of the arguments data.scaled, data. centered, controls some data transformations, and the logical argument common. variance, which, when set to TRUE, considers that all the occasions have the same covariance matrix. These three arguments are discussed in Appendix E. The third set consists of optional arguments which control the function outputs.

\section{Interpretation of fmdsd outputs}

The fmdsd function returns an object of $\mathrm{S3}$ class fmdsd, consisting of a list of 11 elements, including the scores, also called principal coordinates, and the moments of the variables per occasion. The outputs are displayed with the print function, and graphical representations on the principal planes are generated with the plot function.

The interpretation of outputs is based on the relationships between the principal scores and the moments of the densities, in particular their means, variances, covariances, and correlations. These relationships are quantified by correlation coefficients and are represented graphically by plotting the scores against the moments. These interpretation tools are provided by the interpret function, which has two optional arguments: nscores indicating the indices of the column scores to be interpreted and moment whose default value is "mean".

\section{Example}

The following example is treated in detail in Boumaza et al. (2015), using PCA of densities. The data consist of $T=10$ rose bushes assessed three times, by a jury of 14 assessors, for $p=3$ attributes: top-sided shape (Sha), foliage thickness (Den), and plant symmetry (Sym). Here, we present the results obtained with the MDS technique. This presentation is limited to the major steps in the calculation and the visualization of the results generated by the fmdsd, print, plot, and interpret functions. 
$>\operatorname{data}(" r o s e s ")$

$>$ rosesf <- as.folder (roses [,c("Sha", "Den", "Sym", "rose")], groups = "rose")

$>$ resultmds <- fmdsd(rosesf, gaussiand = FALSE, distance $=" 12 "$ )

The function fmdsd displays the barplot of the inertia explained by the first nine principal coordinates (Figure 1).

$>\operatorname{names}($ resultmds)

\begin{tabular}{|c|c|c|c|c|}
\hline [1] "call" & "group" & "variables" "d" & "inertia" & "scores" \\
\hline [7] "means" & "variances" & "correlations" "skewness" & "kurtosis" & \\
\hline
\end{tabular}

By default, the print function applied to resultmds only displays the names of the variables, the inertia, and the principal coordinates.

$>\operatorname{print}($ resultmds)

group variable: rose

variables: Sha Den Sym

$\begin{array}{lrr}\text { inertia } \\ & \text { eigenvalue } & \text { inertia } \\ 1 & 0.02977 & 25.3 \\ 2 & 0.02261 & 19.2 \\ 3 & 0.02028 & 17.2 \\ 4 & 0.01439 & 12.2 \\ 5 & 0.00980 & 8.3 \\ 6 & 0.00930 & 7.9 \\ 7 & 0.00566 & 4.8 \\ 8 & 0.00344 & 2.9 \\ 9 & 0.00262 & 2.2\end{array}$

coordinates

$\begin{array}{llll}\text { rose } & \text { PC. } 1 & \text { PC. } 2 & \text { PC. } 3\end{array}$

$\begin{array}{lllll}\text { A } & \text { A } & 0.055191062 & 0.022167510 & 0.02655143\end{array}$

B $\quad$ B $-0.004963751-0.023764758 \quad 0.07033084$

C C $0.019611171-0.122241048-0.06566866$

D $\quad$ D $-0.091777346 \quad 0.041132410-0.04995275$

E $\quad E-0.013763431 \quad 0.019828288-0.01341398$

F $\quad F \quad 0.016470141-0.024307858 \quad 0.07144865$

G $\quad G-0.088949736 \quad 0.005722199 \quad 0.01223686$

$\mathrm{H} \quad \mathrm{H}-0.025102407-0.0063654740 .01382440$

I I $\quad 0.068203593 \quad 0.043999532-0.02735366$

$\begin{array}{llllll}\mathrm{J} & \mathrm{J} & 0.065080705 & 0.043829197 & -0.03800313\end{array}$

$>\operatorname{plot}($ resultmds)

The output is shown in Figure 2.

$>$ interpret(resultmds)

Pearson correlations between scores and moments

PC. 1 PC. 2 PC. 3

mean. Sha $-0.65 \quad 0.290 .69$

mean.Den $0.83-0.42 \quad 0.08$

mean. Sym $-0.01 \quad 0.94-0.04$

Spearman correlations between scores and moments

PC. 1 PC. 2 PC. 3

mean. Sha $-0.65-0.26 \quad 0.65$

mean.Den $0.78-0.30 \quad 0.02$

mean.Sym $0.16 \quad 0.92-0.35$

The returned plots of the interpret function are not shown. From the correlations between the principal coordinates (PC) and the means of the variables, we deduce that: 
- The higher PC1, the higher "Den", and the lower "Sha" tends to be;

- The higher PC2, the higher "Sym";

- The higher PC3, the higher "Sha" tends to be.

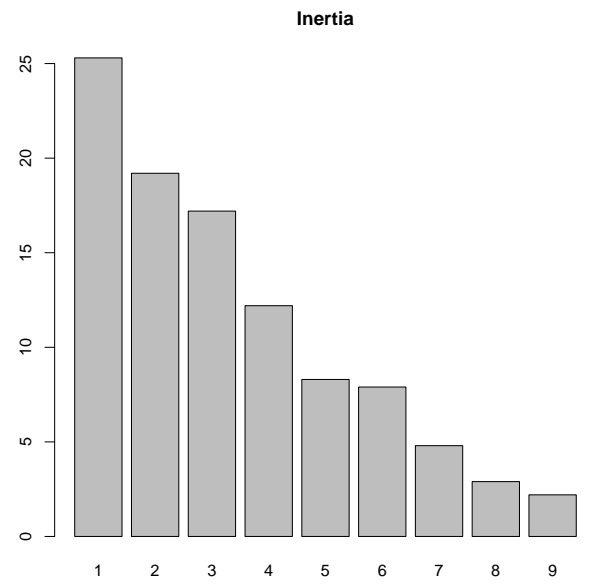

Figure 1: MDS of densities on sensory data (part of the roses data frame): inertia explained by the first ten principal coordinates.
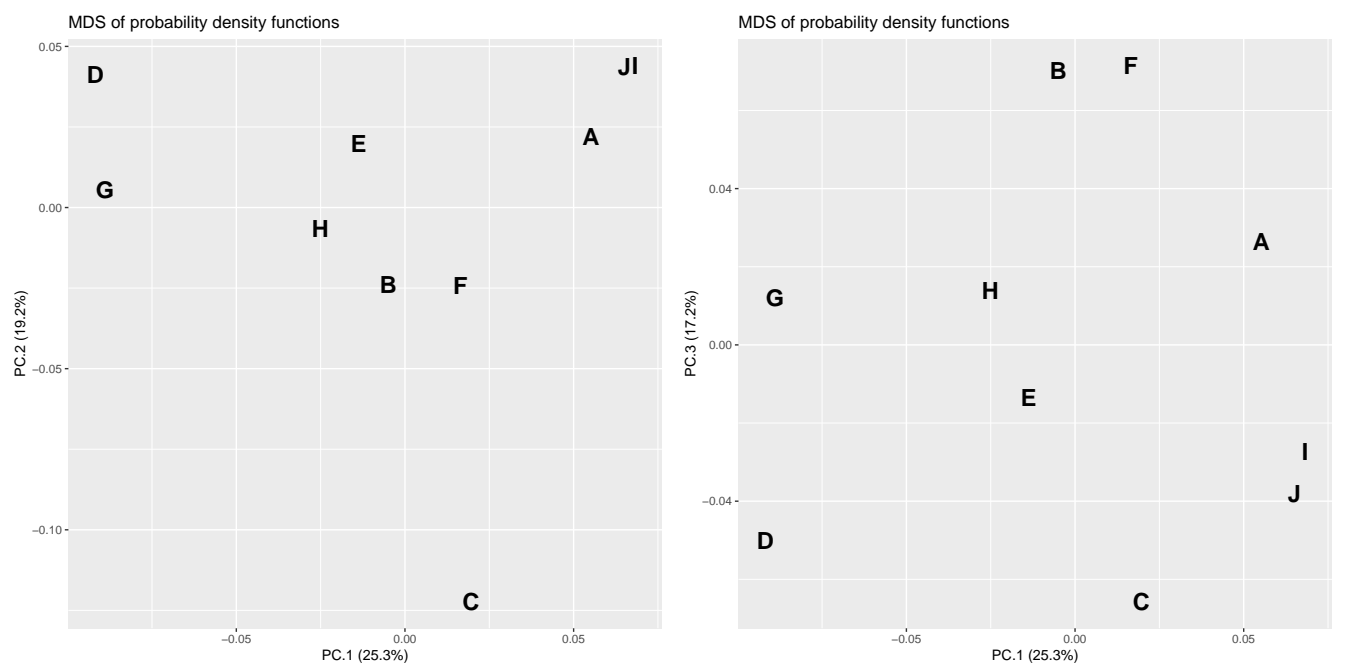

Figure 2: MDS of densities on sensory data (part of roses data frame): the first three principal coordinates.

Thus, from this interpretation of the PCs, we can describe the classes of rose bushes that can be constituted in view of their proximities $v s$. distances visualized in Figure 2. For example, the roses of the class $\{A, I, J\}$ have thick foliage compared to those of the class $\{D, G\}$, the rose bush $C$ is very asymmetrical compared to the other rose bushes, the rose bushes of the class $\{B, F\}$ have a top sided shape.

In order to obtain the correlations between the scores and the standard deviations, we set the optional argument moment to "sd" as in the following example. The other possible values of this argument include "var" (variances), "skewness", "cor" (correlations for multivariate densities).

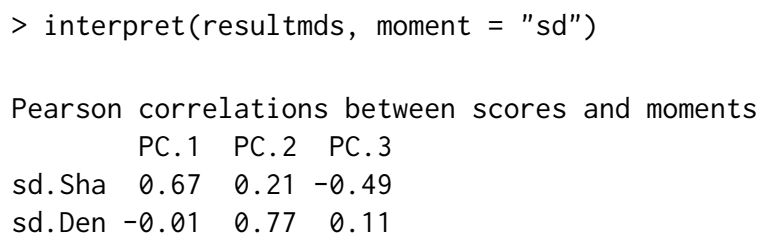


sd. Sym $-0.13-0.44 \quad 0.76$

Spearman correlations between scores and moments

PC. 1 PC. 2 PC. 3

sd. Sha $0.50 \quad 0.45-0.58$

sd.Den $\quad 0.15 \quad 0.64-0.08$

sd.Sym $-0.26-0.76 \quad 0.75$

Some of the correlations between the PCs and the standard deviations of the variables seem high. Reminding that the PCs are related to means, these correlations are therefore clues of links between standard deviations and means of the variables. We, therefore, represent roses using their means and standard deviations (Figure 3). We highlight that the standard deviations/variances used to assess discordance between assessors tend to be smaller when the products subjected to evaluation on a nine-level scale were awarded marks at the ends of the scale. This result which is actually quite intuitive, obtained by the use of MDS on probability density functions, would have been difficult to demonstrate if the means and standard deviations/variances had been analyzed separately.
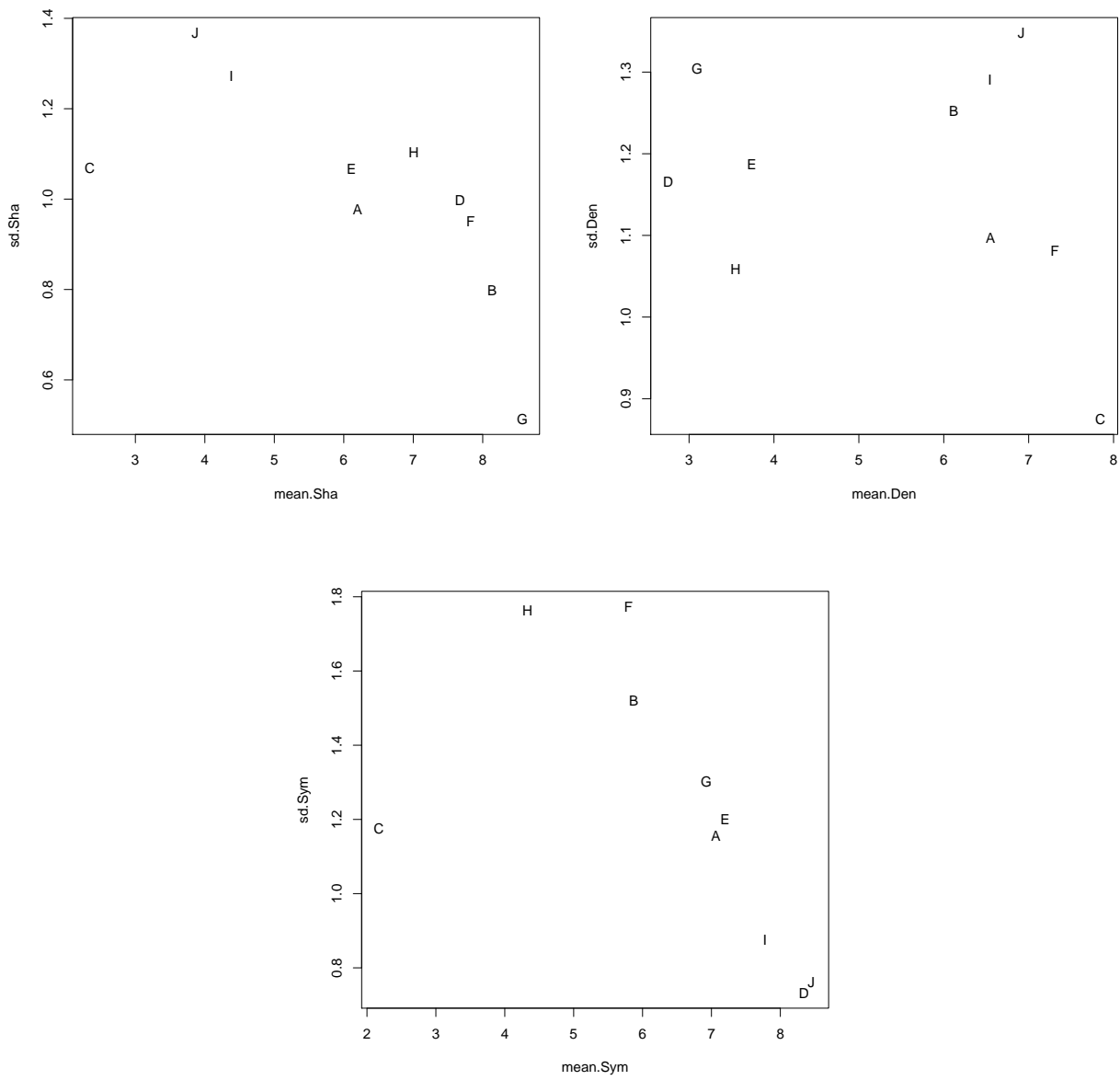

Figure 3: MDS of densities on sensory data (part of roses data frame): relationships between means and standard deviations of the variables.

\section{HCA of densities}

As for MDS, the fhclustd function of the dad package implementing hierarchical cluster analysis of probability density functions is a direct application of hclust, the corresponding function of R. So it is briefly recalled, and we put the emphasis on the interest of coupling the implementation of MDS with HCA in order to interpret more easily the clusters resulting from HCA. 
- Carry out MDS and constitute MDS-classes;

- Specify the characteristics of the MDS-classes according to the moments of the variables;

- Describe HCA-clusters which are similar to MDS-classes;

- Describe the other HCA-clusters by using, when possible, the MDS-scores of the groups making up these clusters.

\section{DA of densities}

With the notations introduced at the beginning of the section Multi-group data: examples and organization, the aim of discriminant analysis of densities (Boumaza, 2004) is to predict the value of $G$ (Table 2) for the occasion $T+1$ represented by the density $f_{T+1}$, knowing $n_{T+1}$ observations of the random vector $X_{T+1}$, which are stored in $\mathbf{X}_{T+1}$ (Table 1).

For each $k=1, \ldots, K$, we denote by $g_{k}$, the density representing the class $k$ of $G$. The predicted value is:

$$
\widehat{k}=\arg \min _{1 \leq k \leq K} D\left(f_{T+1}, g_{k}\right),
$$

where $D$ is a distance index between densities (Distance/divergence between densities section). The data corresponding to the density $g_{k}$ are those corresponding to the $T_{k}$ densities $f_{t}$ belonging to the class $k$ of $G$. In Appendix F, we specify the possible procedures for calculating the densities $g_{k}$ and highlight the link between DA of densities and the linear discriminant analysis (MASS) in the homoscedastic Gaussian case.

The dad package performs all the calculations required to obtain the predicted value through the fdiscd. predict function whose first two arguments, $x$ and class. var, control the input data. It includes the arguments distance and crit, which respectively set the distance and the densities $g_{k}$ (Appendix F). It also includes the arguments gaussiand and windowh, which control the method of density estimation. The fdiscd. predict function returns an object of S3 class fdiscd.predict, which is a list consisting of prior and predicted values corresponding to each $f_{t}$, a confusion matrix, the distances $\left(d_{t k}\right)$ between the $f_{t}{ }^{\prime}$ s and the $g_{k}{ }^{\prime}$ s, and proximities. These are calculated from the inverse of the distances in such a way that their sum is 1 , but they are not probabilities and are useful for a quick comparison of the distances.

In addition, the package calculates the misclassification ratio for the occasions for which the prior class of $G$ is known through the fdiscd. misclass function. This ratio is computed by using the leaveone-out method on the $T$ occasions, and the lower it is, the better the prediction of the variable $G$ by the data $\mathbf{X}$. This function is based on arguments almost identical to those used by the fdiscd.predict function. It also generates similar outputs, grouped into an object of the $\mathrm{S} 3$ class fdiscd.misclass. However, these two functions differ in the method used to calculate the distances $\left(d_{t k}\right)$ : if $f_{t}$ belongs to the $k$-th class of $G$, the data corresponding to $f_{t}$ are not included in the data used to estimate $g_{k}$. This function is useful for empirical investigations in order to identify the optimal values of the arguments minimizing the misclassification ratio. These values are then used by the fdiscd. predict function for prediction of the $G$ class of an occasion of unknown class.

\section{First example: Castles / Stones}

Let us consider the archaeological data (Section Multi-group data: examples and organization, Example 1). For the sake of clarity, we combine the six classes to yield three final classes, 1140-1175, 1175-1280, and 1280-1550, numbered 1 to 3.

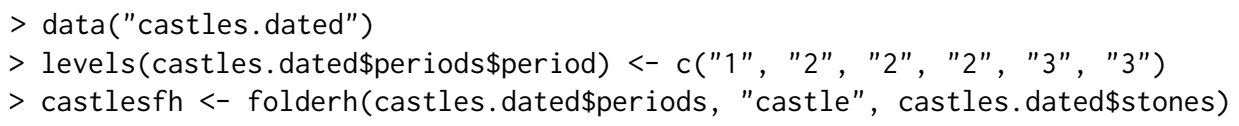

The fdiscd.misclass function is used to calculate the misclassification ratios (global and per class).

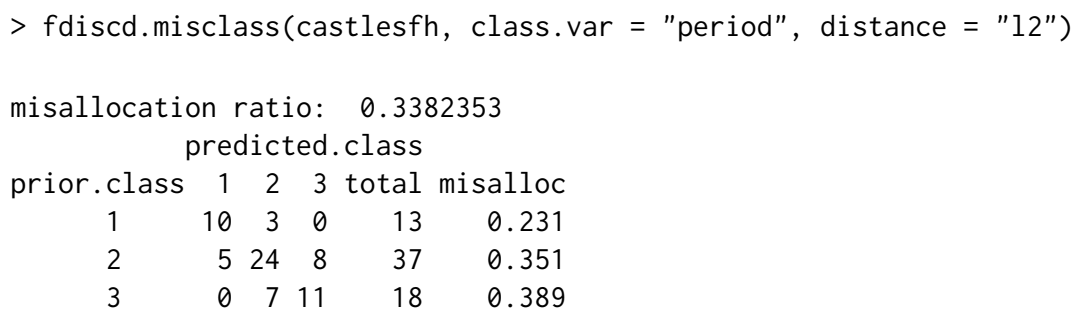




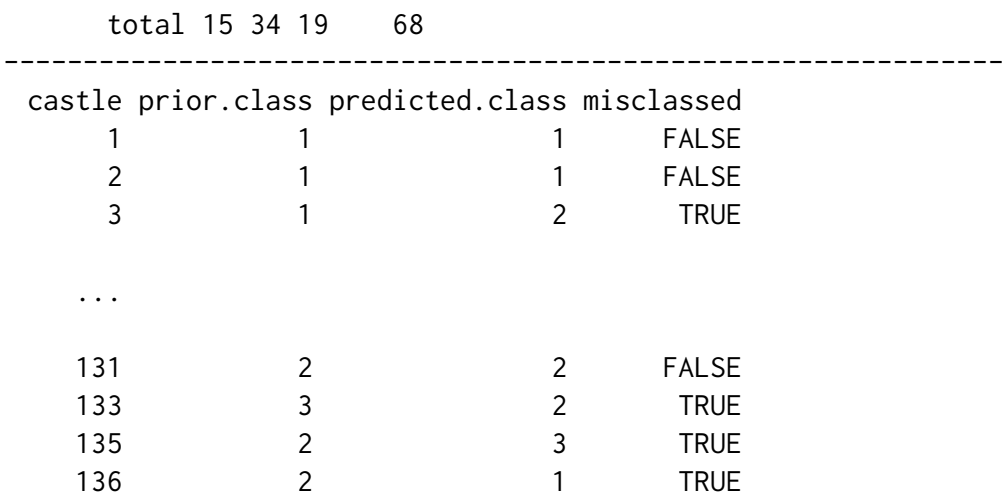

In order to empirically calibrate the different arguments of the function, the previous operation is repeated, choosing other values for the arguments. If the gaussiand argument is set to TRUE, the smallest global misclassification ratio is $34 \%$ (Table 6) and is obtained for crit $=1$, the default value for this argument, and for distance = "12" or "hellinger".

Estimating the densities by the Gaussian kernel method by setting gaussiand = FALSE, with the bandwidth defined by (2) and (3), increased the global misclassification ratio to $40 \%$. If we consider the same proportionality coefficient $h$ in the formula (2), that is

$$
\mathbf{h}_{t}=h \widehat{\mathbf{V}}_{t}^{1 / 2},
$$

by setting the argument windowh of the function fdiscd.misclass, we empirically obtain a value of $h$ that minimizes the misclassification ratio. For this purpose, we calculate the misclassification ratio for different values of windowh (Table 7). Note that an optimal empirical value is about windowh $=0.6$, with a misclassification ratio of $32 \%$. One of the best empirical parametrizations of the fdiscd. predict function would be gaussiand $=$ FALSE, windowh $=0.6$, and crit $=1$.

\begin{tabular}{l|ccc} 
crit & 1 & 2 & 3 \\
\hline Ratio & 0.34 & 0.56 & 0.40
\end{tabular}

Table 6: Castles/Stones. Misclassification ratio assuming densities to be Gaussian and parametrically estimated, depending on the value of the crit argument used to select the type of $g_{k}$ density (Appendix F).

\begin{tabular}{l|ccccccccc} 
windowh & 0.1 & 0.2 & 0.3 & 0.4 & 0.5 & 0.6 & 0.7 & 0.8 & 0.9 \\
\hline Ratio & 0.56 & 0.44 & 0.38 & 0.38 & 0.37 & 0.32 & 0.32 & 0.35 & 0.34
\end{tabular}

Table 7: Castles/Stones. Misclassification ratio for densities estimated by the Gaussian kernel method, depending on the value of the windowh argument, the proportionality coefficient setting the bandwidth.

The misclassification ratios are disappointing. Thus, the method was used only as an indication to date the castles and its results were cross-checked with other established facts from other historical sources (Rudrauf and Boumaza, 2001).

\section{Second example: Rosebushes/Leaves}

Let us consider the agronomic data (Section Multi-group data: examples and organization, Example 2). We consider only the continuous variables characterizing the leaves, that is, leaving out the discrete variable "number of leaflets". As in the first example, we carry out discriminant analysis by applying the fdiscd.misclass function for different parameter values. Table 8 gives the classification error rates for the three criteria and some values of the proportionality parameter. One of the best combinations of parameter values would, therefore, be crit $=3$ and windowh $=0.2$. The error rate is 0.032 , which is clearly better than the result obtained with the archaeological data of the first example. If the same method is applied to all variables, including "number of leaflets", the error percentage is zero for crit $=3$ and windowh $=0.3$.

In these two previous examples, it should be noted that the search for an optimum value of the parameter windowh has the disadvantage of being carried out by trial and error and is a time-consuming procedure. 


\begin{tabular}{|c|c|c|c|}
\hline windowh & crit = 1 & crit = 2 & crit $=3$ \\
\hline 0.1 & 0.742 & 0.806 & 0.129 \\
0.2 & 0.452 & 0.677 & $\mathbf{0 . 0 3 2}$ \\
0.3 & 0.226 & 0.452 & 0.097 \\
0.4 & 0.129 & 0.226 & 0.097 \\
0.5 & 0.097 & 0.129 & 0.161 \\
0.6 & 0.097 & 0.129 & 0.161 \\
0.7 & 0.065 & 0.129 & 0.194 \\
0.8 & 0.065 & 0.097 & 0.226 \\
0.9 & 0.065 & 0.097 & 0.226 \\
\hline
\end{tabular}

Table 8: Rosebushes/Leaves. Misclassification ratio for the three criteria and different values of the proportionality parameter windowh, the densities being estimated by the Gaussian kernel method.

\section{Practical advice}

\section{Data management}

The functions folder and as. folder play a central role in the creation of objects handled by the functions fmdsd (MDS on continuous data), mdsdd (MDS on discrete data), fhclustd (HCA on continuous data), and hclustdd (HCA on discrete data). The functions folderh and as. folderh play the same role in DA context: fdiscd.misclass and fdiscd. predict in the continuous case, or discdd.misclass and discdd. predict in the discrete case.

It is advisable for the user to know how to use them, especially as their handling is quick and easy (Appendix C).

\section{Computation times}

The computation times of the functions of dad depend mainly on the computation time of the distances between groups in MDS and HCA contexts or between groups and classes in the DA context. The longest times are achieved when the densities are estimated by the kernel method (Calculation of $L^{2}$ distances between continuous non-Gaussian densities estimated by the kernel method section). When applying the function matdisl2 with the option method = "kern" on the archeological data (Example 1 of Appendix G), the computation time is approximately five times greater than when method = "gaussiand".

Among the main functions of dad, the most time-consuming one is undoubtedly fdiscd.misclass when its gaussiand argument is set to FALSE. That is, the densities are non parametrically estimated. For example, applying this function to archaeological data with gaussiand = TRUE takes less than one second while it takes approximately 30-40 seconds with gaussiand = FALSE (Example 2 of Appendix G). Thus, we recommend doing tests on small datasets when working on non-Gaussian continuous data.

\section{Choice of distance}

The choice of a distance index depends above all on the modeling hypotheses: discrete or continuous data. If they are discrete dad proposes five indices (Table 4). If they are continuous, it proposes five indices in the Gaussian case (Table 5) and only one for non-Gaussian data, which is the $L^{2}$ distance combined with the estimation of the densities by the Gaussian kernel method.

The distance indices of discrete or Gaussian densities are compared on particular examples in the context of HCA (Appendix A) or DA (Appendix B). It is shown that depending on the technique used and the criterion for measuring the quality of the results, a distance index can be better than another for a set of data and worse for another set. Thus, in the discrete or Gaussian cases, if there is no a priori choice of distance index, we suggest an empirical approach. As the computation times are reasonable, we perform the desired analysis by experimenting with each of the distance indices, then choose the distance index according to one of the two criteria given by dad.

For DA, it is the misclassification ratio obtained by the one-leave-out procedure by using the function fdiscd.misclass. We should choose the distance index which gives the lowest misclassification ratio.

For MDS, it is the interpretation of principal coordinates in terms of marginal distributions (discrete 
densities) or moments (continuous densities) using the function interpret. We should choose the distance index which gives the highest correlations between principal coordinates and marginal distributions or moments. For HCA, we combine it with the MDS method (HCA and MDS on the same densities subsection) and, therefore, we refer to the above to choose the distance index.

\section{Discussion and concluding remarks}

The classic statistical methods as multidimensional scaling (MDS), hierarchical clustering analysis (HCA), and discriminant analysis (DA) operate on data which are rows of a data frame. They are available in the R packages stats, MASS (Venables and Ripley, 2002), ade4 (Dray et al., 2007), FactoMineR (Lê et al., 2008), cluster (Maechler et al., 2019). The dad package presented in the manuscript generalizes them to data that are organized into groups or occasions. The graphics produced by MDS, the clusters constituted by HCA, or the predicted class provided by DA, concern the occasions and not the individuals who constitute them.

These methods are multivariate data analysis, but they can also be seen as functional data analysis since they operate on multivariate functions estimated from multivariate data. They are theoretically similar to some functional statistical methods implemented in fda.usc, fda, and fdadensity packages. The main difference is in the type of data processed. The functional statistical methods deal with functions of only one or two variables, while the main functions of dad can deal with more.

These methods can also be seen as compositional data analysis. Indeed, compositional dataset provides portions of the total (Aitchison, 1986) and the R packages compositions, Compositional, and robCompositions are devoted to such data by implementing many descriptive or graphical techniques and models. Regarding the packages Compositional and robCompositions, their modeling approach is far enough from our approach to be addressed in this discussion, while the package compositions presents similarities with our work which we detail in Appendix H. In the case of a univariate discrete density, the differences concern the method of interpreting the graphical outputs provided by princomp of compositions and mdsdd of dad even if we get fairly similar graphics (see the example of Appendix H). In the multivariate case, dad takes into account marginal distributions of orders 1 and 2 (or more), while in the current version of compositions, only marginal distributions of order 1 are taken into account.

The most recent version of the dad package implements functions which operate on discrete data. It also extends the three previous methods to a mixture of numerical and categorical data by transforming the numerical data into categorical data. It is done by dividing the range of each numerical variable into intervals using the function cut. folder, an extension of the function cut to several variables of a folder.

In addition, the last versions of functions implementing MDS and HCA apply to data stored in a data frame and not only to data stored in a folder.

The following development work is also planned:

- Automation of the empirical search for the proposed proportionality coefficient in the formula (5), considered optimal (in at least some ways) in the context of discriminant analysis;

- Automation of the empirical search for a similar coefficient suitable for use in multidimensional scaling as proposed by Yousfi et al. (2015) in principal component analysis context.

\section{Summary}

When we work on multidimensional multi-group data and are interested in the groups and not in the individuals who make up these groups, we want to have statistical methods and computer tools making it possible to describe these groups. The dad package is devoted to this.

It mainly provides elaborate functions which implement multivariate techniques such as multidimensional scaling, hierarchical classification analysis, or discriminant analysis on such groups. Moreover, in order to help users in reading the outputs of these techniques, dad provides functions for interpreting the results.

It provides datasets which illustrate such data and easy-to-use functions which allow to (i) manage multi-group data by associating a data frame with each group, (ii) compute the distances between groups based on the mathematical concept of probability distribution/density, and (iii) secondarily compute elementary statistics by group as for example frequency distributions and moments.

It defines new data structures called folders (folder, folderh, foldermtg) and provides specific tools to manage them, such as selecting or deleting columns from a folder, converting numeric 
columns of a folder to factors. The most noticeable among them allows to easily import into R plant architectures encoded in $\mathrm{mtg}$ files and, thus, have R packages available to analyze the imported data.

\section{Acknowlegments}

The authors thank Gilles Hunault and Julie Bourbeillon, the Associate Editor, and the reviewers for their valuable comments and contributions to this work.

\section{Appendix A: HCA and distance index}

We compare three distance indices in the HCA context with a small, simple example originating from an exchange with an anonymous reviewer of a previous version of the manuscript by comparing the $L 1$ and $L 2$ distances and the symmetrized divergence of Kullback-Leibler. In his review, he made a severe criticism of the $L 2$ distance, which was the unique distance proposed in the first versions of the dad package: "Let $f$ a uniform density in the interval [0, 1], and g also uniform in [0, 0.90]. Clearly, from the point of view of $g$, it is impossible to reach values in the interval $(0.90,1]$ and so, these two densities are not neighbors." Indeed, the $L 1$ and $L 2$ distances and the symmetrized Kullback-Leibler divergence (KL) are:

$$
L 1(f, g)=0.20, L 2(f, g)=0.33, K L(f, g)=\infty,
$$

so $K L(f, g)$ reflects this impossibility of reaching all the values of $f$ from $g$, unlike $L 1$ and $L 2$, which consider them to be relatively close to each other.

However, if we add the other two densities $v$ and $w$, uniform on $[0,0.10]$ and $[0,0.11]$, and if our objective is to obtain either a partition of $f, g, v$, and $w$ in 2 classes, or an approximate representation on a plane of these 4 densities, the choice of $L 1$ or $L 2$ is more informative than $K L$ (Table 9) because with the first two distances, we would easily group $f$ and $g$ on one side and $v$ and $w$ on the other side, whereas with KL all distances are infinite. Our belief is that the choice of a distance between

\begin{tabular}{|ccccc|ccccc|ccccc|}
\hline L1 & & & & & L2 & & & & & KL & & & \\
\hline & $f$ & $g$ & $v$ & $w$ & & $f$ & $g$ & $v$ & $w$ & & $f$ & $g$ & $v$ & $w$ \\
$f$ & 0 & 0.20 & 1.80 & 1.78 & $f$ & 0 & 0.33 & 3.00 & 2.84 & $f$ & 0 & $\infty$ & $\infty$ & $\infty$ \\
$g$ & & 0 & 1.78 & 1.76 & $g$ & & 0 & 2.98 & 2.82 & $g$ & & 0 & $\infty$ & $\infty$ \\
$v$ & & & 0 & 0.18 & $v$ & & & 0 & 0.95 & $v$ & & & 0 & $\infty$ \\
$w$ & & & & 0 & $w$ & & & & 0 & $w$ & & & & 0 \\
\hline
\end{tabular}

Table 9: $L 1, L 2$, and symmetrized Kullback-Leibler distance/divergence between the uniform probability densities $f=U([0,1]), g=U([0,0.9]), v=U([0,0.10])$, and $w=U([0,0.11])$.

groups cannot be made in the absolute. It is better to specify a criterion for choosing the distance, which makes sense in the context of the method used, such as percentage of inertia explained in MDS (Delicado (2011)), or in the context of the data analysed. In the Practical advice section, we suggest two other criteria.

\section{Appendix B: DA and distance index}

In the two examples of $D A$ of densities section, the data were not considered plausibly Gaussian and the distance used is the $L^{2}$ distance, the only distance computable in dad which is suitable for this type of data. In this appendix, we are interested in other types of data and in the misclassification ratios according to the distance index used (Distance/divergence between densities section).

\section{Simulated discrete data}

Let $p_{1}=\mathcal{P}\left(\lambda_{1}\right)$ and $p_{2}=\mathcal{P}\left(\lambda_{2}\right)$ be two Poisson distributions with respective parameter $\lambda_{1}=1$ and $\lambda_{2}=2$ and $S_{1}$ and $S_{2}$ respective simulated samples of size 30 . We simulate a sample $S$ of size 10 according to the distribution $p_{1}$, we calculate the dissimilarities $d_{1}$ and $d_{2}$ between this sample and each of the samples $S_{1}$ and $S_{2}$, then we compare $d_{1}$ and $d_{2}$. We would expect $d_{1}$ to be less than $d_{2}$ since the samples $S$ and $S_{1}$ are from the same population. Thus, if $d_{1}>d_{2}$ we consider that the sample $S$ is misclassified. We repeat the previous scenario 1000 times and then calculate the misclassification ratio. 
The results obtained for the same 1000 samples and each of the distance indices of Table 4 are in column (a) of Table 10. Then we proceed in the same way with 1000 samples of the distribution $p_{2}$; the misclassification ratios are in column (b). We notice that for the Jeffreys divergence, the number of

\begin{tabular}{lll}
\hline Distance index & $(\mathrm{a})$ & $(\mathrm{b})$ \\
& $\begin{array}{l}\text { Misclassification ratio } \\
\text { of } 1000 \text { samples from } p_{1}\end{array}$ & $\begin{array}{l}\text { Misclassification ratio } \\
\text { of 1000 samples from } p_{2}\end{array}$ \\
\hline Symmetric chi-square & 0.073 & 0.183 \\
Hellinger & 0.064 & 0.188 \\
Jeffreys & $0.049\left(600^{*}\right)$ & $0.139\left(643^{*}\right)$ \\
Jensen-Shannon & 0.139 & 0.269 \\
Lp & 0.079 & 0.172 \\
\hline & $\left(^{*}\right)$ number of samples $S$ at infinite distance from samples $S_{1}$ or $S_{2}$.
\end{tabular}

Table 10: Poisson distributions. Misclassification ratios of 1000 samples from $p_{1}=\mathcal{P}_{1}$ (a) or from $p_{2}=\mathcal{P}_{2}(\mathrm{~b})$. The instructions for $\mathrm{R}$ allowing the calculation of the ratios corresponding to the symmetric chi-square index are given in the last subsection. The other ratios are obtained by means of the corresponding distance while keeping the same simulation seed.

samples $S$ at infinite distances from $S_{1}$ or $S_{2}$ is very large, which makes it inefficient at discriminating between samples.

We carried out several simulations as in the previous procedure to compare the distances. We did not find any stability in the order of distances according to the misclassification ratios.

\section{Simulated Gaussian data}

As for the discrete case, these distance indices are compared in a simplified context of discriminant analysis using the same procedure. Let $p_{1}$ and $p_{2}$ be the two Gaussian distributions $N(0,1)$ and $N(1,4)$. The results are given in Table 11 . We notice that for the samples from $N(0,1)$ (a), the distance

\begin{tabular}{lll}
\hline Distance index & $(\mathrm{a})$ & $(\mathrm{b})$ \\
& $\begin{array}{l}\text { Misclassification ratio } \\
\text { for 1000 samples from } p_{1}\end{array}$ & $\begin{array}{l}\text { Misclassification ratio } \\
\text { for 1000 samples from } p_{2}\end{array}$ \\
\hline Hellinger & 0.020 & 0.051 \\
Jeffreys & 0.018 & 0.043 \\
L2 & 0.034 & 0.040 \\
L2N & 0.026 & 0.111 \\
2-Wasserstein & 0.007 & 0.169 \\
\hline
\end{tabular}

Table 11: Gaussian distributions. Misclassification ratios of 1000 samples from $p_{1}=N(0,1)$ (a) or from $p_{2}=N(1,4)(\mathrm{b})$. The $\mathrm{R}$ instructions are similar to those of Poisson samples. It suffices to change the function rpoi to rnorm with the appropriate parameters. In these simulations, the seed is set to 123.

giving the best rate is the Wasserstein distance. On the other hand, it is the worst for the samples from $N(1,4)(b)$.

We carried out several simulations and the conclusion is word for word the same as that of the discrete case with the Poisson distributions.

These results lead us to suggest choosing a distance index based on the minimum misclassification ratio in the subsection Choice of distance of Practical advice Section.

\section{Computation of the misclassification ratios of Table 10 or 11}

The following $\mathrm{R}$ instructions are used to compute the misclassification ratios in DA context with samples from Poisson distributions according to the symmetric chi-square distance index of Table 10. The instructions for computing the other ratios of Table 10 and the ratios of Table 11 are given in supplementary material.

$>n<-30$
$>$ ne $<-10$ 


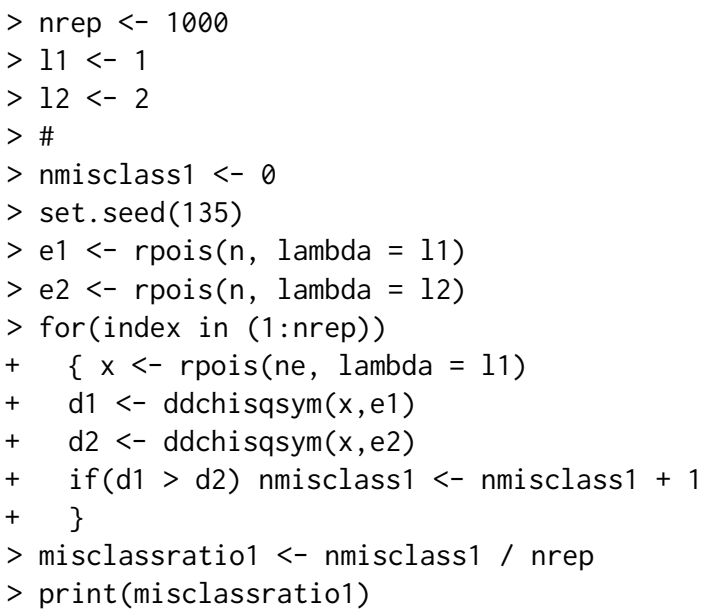

[1] 0.073

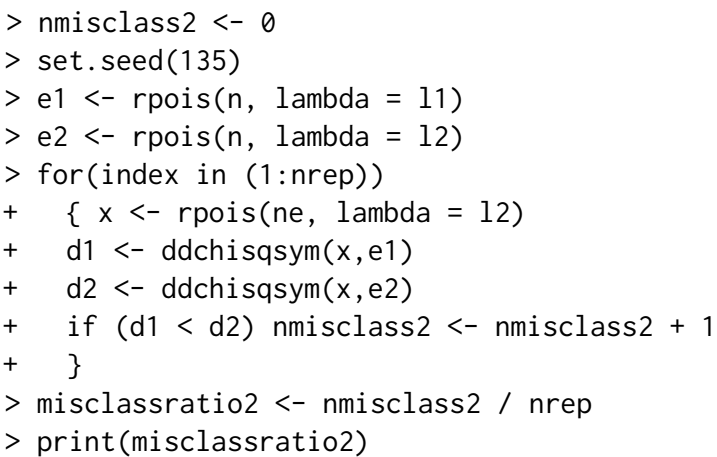

[1] 0.183

\section{Appendix C: some useful functions on data folders}

The dad package uses objects of class folder or folderh. These objects are lists of data frames having particular formats.

\section{Introductory example}

Let us consider the archaeological data introduced in Multi-group data: examples and organization section. The data that Jean Michel Rudrauf (Rudrauf and Boumaza, 2001) submitted to us was in the form of a paper binder, each sheet of which corresponds to a castle (Figure 5). So for each castle we have:

- a name,

- an identification number,

- a building period (sometimes even a year) if it is known,

- the $\mathrm{p}=4$ measurements of a sample of stones.

A most natural and least redundant way for entering such data is to create:

- one data frame per castle. This data frame with $\mathrm{p}$ columns is named by the identification number of the castle and contains the measurements of the stones. Its number of rows corresponds to the number of stones whose all measurements are available.

- a data frame whose rows are the castles and whose columns correspond to the name, the identifier and the building period.

From there, we choose to suggest suitable data structures for multi-group data and propose management and calculation tools adapted to these data structures. This is exemplified below.

The archaeological data are stored in the list castles.dated of two data frames. The data frame castles. dated\$periods consists of $T=68$ rows (castles) and 2 columns: castle, the castle 


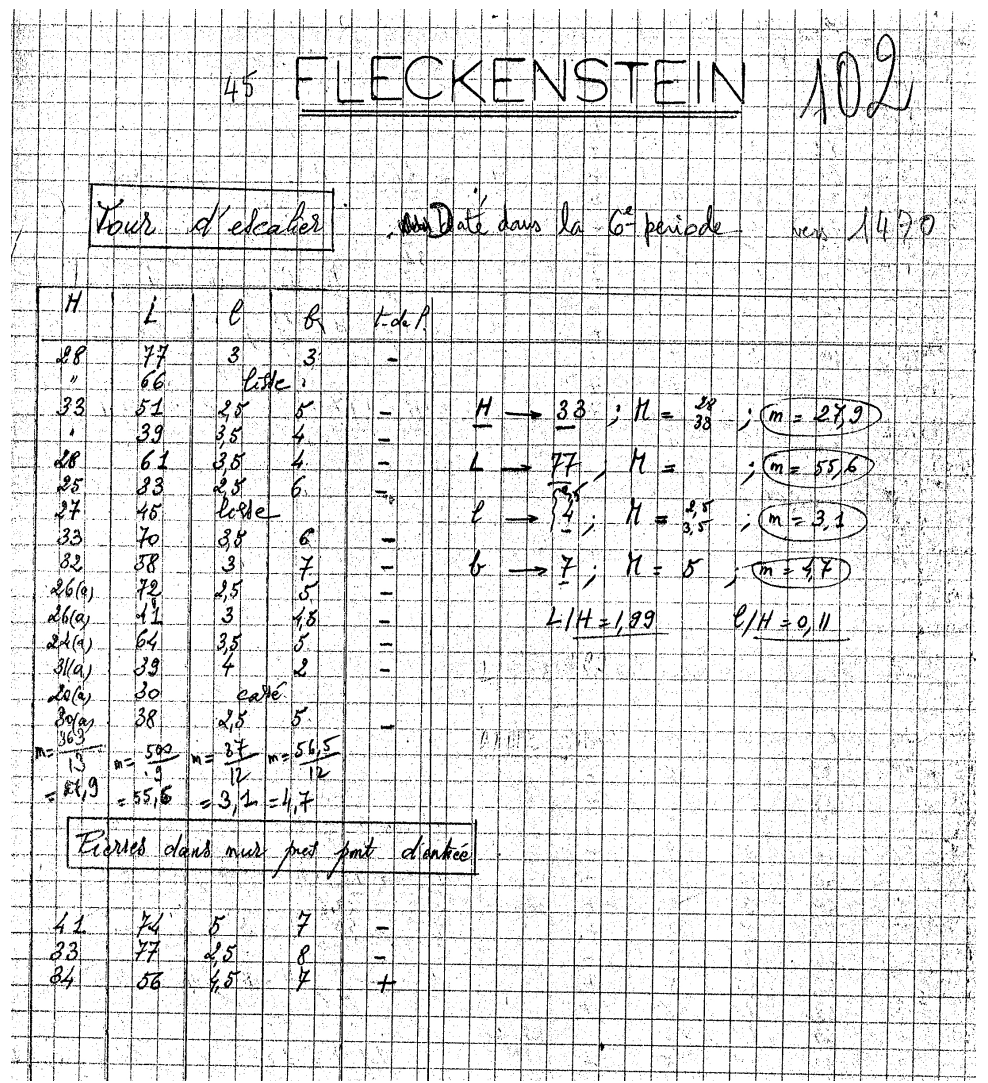

Figure 5: The data patiently collected by Jean Michel Rudrauf corresponds to the castle of Fleckenstein. Its number identification is 102. It was built around 1470 i.e. in the sixth period. The measurements are $\mathrm{H}$ : height, L: width, 1 : edging and $\mathrm{b}$ : boss. There are 10 stones whose measurements are complete: 7 stones belong to the staircase tower (Tour d'escalier) and 3 stones belong to a wall located near the entrance of the castle (Pierres dans mur près pont d'entrée). The corner stones designated by the symbol (a) in column $\mathrm{H}$, are excluded from the study.

identifier, and period, the building period which is a factor with 6 levels. The second data frame castles. dated\$stones consists of 1262 rows (stones) and 5 columns: 4 numeric characteristics of the stones (height, width, edging, and boss) and 1 factor castle with 68 levels, which gives the identifier of the castel to which belongs each stone. The implementation of DA requires carrying out calculations not only on all the stones of each castle but also on all the stones of all the castles of each period. To do this, we can store the stones in a data frame made up of 6 columns obtained by adding a column period to the file castles. dated\$stones. Thus, all the stones of a castle have the same period value. In order to avoid this redundancy in data entry and management, we propose to store the two files castles.dated\$stones and castles. dated\$periods as well as the key castle which relates them, in a folderh that is a list of two data frames related by a key. The procedure for doing this is given in the paragraph First example: Castles/Stones of DA of densities section.

Now consider only the castles. dated\$stones file. To implement both DA and MDS, it is necessary first to calculate and store the vectors of means and the covariance matrices of the $p=4$ numeric variables for each castle, then to use the results in the calculation of the distances between each pair of castles. The calculations of means and covariances by castle can be carried out directly from the five-column data frame castles. dated\$stones using, for example, the following $\mathrm{R}$ functions: colMeans, var, and by. In order to facilitate the extraction of data relating to a particular castle and control the data entry, or to check pieces of the computer program, we organize the data in a folder that is a list of $T$ four-column data frames (one data frame per castle), and we extend the mean and var functions so that they apply to such a data structure and return results as lists. So if $x$ is the name of the folder, $x[[t]]$ is the data frame which contains the data of the castle $t$, mean $(x)[[t]]$ is its vector of means and $\operatorname{var}(x)[t]]$ is its covariance matrix. We find that by doing so, it is also easier to remember the names and contents of these objects when writing computer programs. 


\section{Objects of class folder}

Such objects, lists of data frames which have the same column names, are created by the folder function, from two (or more) data frames, say $x 1$ and $\times 2$, as follows: folder $(x 1, x 2)$.

Optional argument cols. select defines the way in which the columns of the two data frames are selected: common columns only (cols. select = "intersect") or all the columns (cols. select = "union"). For the "union" option, if the data frames do not have exactly the same column names they are complemented by NA's.

The functions mean. folder (or simply mean), skewness. folder, and kurtosis. folder applied to the object $x$ of the class folder, respectively return the list of the vectors of means, skewnesses, and kurtosises of the numeric columns of the elements of $x$. The functions var. folder and cor. folder return the list of the covariance and correlation matrices. If the data frames of $x$ contain non-numeric columns, these functions exclude these columns from the computation. If they contain only one numeric column, these functions return lists of numbers. Note that map ( $x, \operatorname{colMeans}), \operatorname{map}(x, \operatorname{cor})$, and $\operatorname{map}(x, v a r)$ return the same values as before, except in the presence of a factor column whose levels are numbers: $\operatorname{map}(x, \operatorname{colMeans})$ and $\operatorname{map}(x, c o r)$ return an error while map $(x, v a r)$ integrates the factor column in the covariance matrices.

Hence, in folder objects, the observed variables are the same on every occasion, unlike individuals which can be different from one occasion to the next. The particular case where individuals are the same on every occasion, corresponds to data defined as three-way data by Kiers (2000) in his essay on standardization of terminology for multiway analysis. In this particular case, it would be better to store the data in an object of class array.

\section{Objects of class folderh}

Such objects are hierarchical lists of data frames in which two successive data frames from the list are related by means of a key. We complete the presentation of the introductory example by a case with more than two data frames. For three data frames, say df1, df2, and df3, there are two keys: the first, say key1, describes the " 1 to $N$ " relationship between $\mathrm{df} 1$ and $\mathrm{df} 2$, and the second, say key2, describes the " 1 to $N$ " relationship between $\mathrm{df} 2$ and $\mathrm{df} 3$. The arguments of the folderh function are introduced in the following order: $d f 1, k e y 1, d f 2, k e y 2, d f 3$, and so on, if there are more than three data frames. An example of such object is given in Appendix D.

The function as.data.frame applied to such a hierarchical folder, say fh, whose constituent elements are listed above, has two main arguments: key (the name of a key of fh) and elt (the name of a data frame of $\mathrm{fh}$ ) with the precision that the value of elt is located after the value of key in the list of arguments defining $\mathrm{fh}$. In the case of two adjacent names, that is key = key 1 and elt $=\mathrm{df} 2$ or key $=$ key 2 and $e l t=d f 3$, as. data. frame returns a data frame similar to any viewpoint to that returned by the merge function. If key $=k e y 1$ and $e l t=d f 3$, the data frame returned by the as.data.frame function, say $d f r$, has the same rows as df3. The columns of $d f r$ are those of the data frames $d f 3$ and $d f 1$, and those corresponding to all the keys located between key 1 and $d f 3$ in the list defining fh, noticing that the key columns are the first columns of $d f r$.

\section{Appendix D: Import plant architectures encoded in $\mathrm{mtg}$ files}

The result of this import procedure consists of an object of class folderh and uses an intermediate object of class foldermtg. Let us first specify what an $\mathrm{mtg}$ file is.

The topological structure of a plant is defined from its decomposition into elementary components and the connections between them (Godin and Caraglio, 1998). In Figure 6, the plant is composed of 2 axes (one principal axis, A1, coming from the root and bearing one secondary axis, A2) and each axis is composed of internodes and peduncles: the principal axis is composed of seven internodes I1, .., I7 and one peduncle F1 and the secondary axis is composed of three internodes I8, I9 and I10. Among the computer file types used to store the topology of a plant, we are interested in mtg (multiscale tree graph) files which can be opened with a spreadsheet as LibreOffice-Calc, or Excel (Pradal and Cokelaer, 2010). Breaking a plant into axes and breaking each axis into internodes create two " 1 to $N$ " relationships which can be stored in an object of class folderh. This hierarchical folder, say fh, is a list of three data frames P (set of 1 plant), A (set of 2 axes), and I (set of 10 internodes), and two keys $P$ and $A$. It is the result of the following three $R$ instructions which successively imports an $\mathrm{mtg}$ file into $\mathrm{R}$, creates an object of the $\mathrm{S} 3$ class foldermtg, and then creates fh.

$>$ mtgfile <- system.file("extdata/plant2.mtg", package = "dad")

$>x 2<-$ read.mtg(mtgfile) 


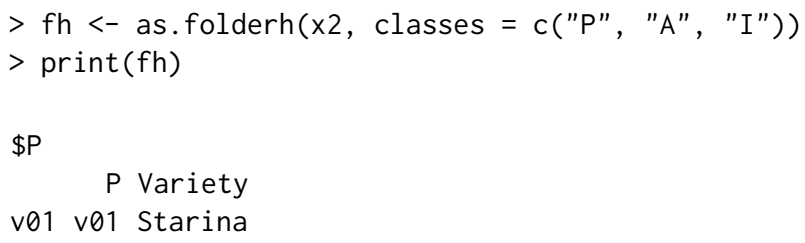

$\$ A$

$\begin{array}{rrrr} & P & A & \text { Length } \\ \text { v02 } & \text { v01 } & \text { v02 } & 30 \\ \text { v07 } & \text { v01 } & \text { v07 } & 12\end{array}$

$\$ I$

A I Leaflet

v03 v02 v03 3

$v 04$ v02 $v 04 \quad 3$

v05 v02 $v 05 \quad 5$

v06 v02 v06 5

$\begin{array}{llll}\vee & \vee 08 & \vee 08 & 5\end{array}$

v09 $\vee 07$ v09 5

$\vee 10 \vee 07 \vee 10 \quad 7$

v11 $\vee 02$ v11 7

v12 $\vee 02 \vee 12 \quad 5$

v13 v02 v13

$\operatorname{attr}(, "$ class")

[1] "folderh"

attr (, "keys")

"P" "A"

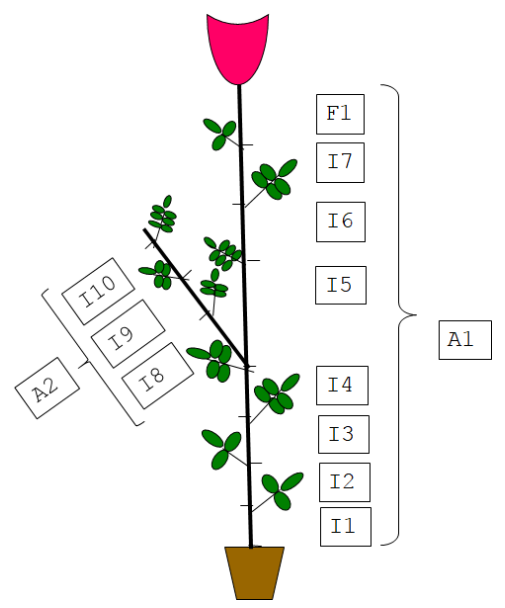

Figure 6: Decomposition of a plant $(P)$ into axes $(A)$, internodes $(I)$, and peduncles $(F)$.

\begin{tabular}{|c|c|c|}
\hline \multirow{2}{*}{\multicolumn{2}{|c|}{$\begin{array}{l}\text { Biological component } \\
\text { /P1 }\end{array}$}} & File code \\
\hline & & v01 \\
\hline$\wedge / \mathrm{A} 1$ & & v02 \\
\hline$\wedge / \mathrm{I} 1$ & & v03 \\
\hline$\wedge<\mathrm{I} 2$ & & v04 \\
\hline$\wedge<\mathrm{I} 3$ & & v05 \\
\hline$\wedge<\mathrm{I} 4$ & & v06 \\
\hline & $+\mathrm{A} 2$ & v07 \\
\hline & $\wedge / \mathrm{I} 8$ & v08 \\
\hline & $\wedge<$ I9 & v09 \\
\hline & $\wedge<\mathrm{I} 10$ & v10 \\
\hline$\wedge<\mathrm{I} 5$ & & v11 \\
\hline$\wedge<\mathrm{I} 6$ & & v12 \\
\hline$\wedge<\mathrm{I} 7$ & & v13 \\
\hline$\wedge<\mathrm{F} 1$ & & v14 \\
\hline
\end{tabular}

Table 12: The first two columns correspond to the plant topology stored in an $\mathrm{mtg}$ file. The third column corresponds to the code of each component: the plant $(\mathrm{P})$ is encrypted $\mathrm{v} 01$, etc.

An object of class foldermtg is a list of data frames. It is only an intermediary to which the only function of $\mathrm{R}$ which can be applied to it is as. folderh. This, therefore, makes it possible to retrieve a hierarchical folder which consists of data frames, one data frame per type of biological components, on which one can operate statistical calculations by means of $\mathrm{R}$ functions.

\section{Appendix E: Particular cases of the fmdsd function}

We assume that the densities associated with the occasions are Gaussian. For particular values of the arguments data. centered, data.scaled, and common. variance, the outputs of the fmdsd function are similar to those returned by other functions. 
common. variance $=$ TRUE

The Gaussian densities are assumed with the same covariance matrix which is estimated using all data. Thus, the distances between the densities are reduced to the differences between their mean vectors (Table 5). The Euclidian distances between mean vectors computed by the $\mathrm{R}$ function dist are equal to that computed by the 2-Wasserstein distance between densities. In this case, the R function cmdscale applied on the mean vectors returns exactly the outputs of the fmdsd function.

data . centered $=$ TRUE

The Gaussian densities are assumed with zero mean vectors. Consequently, the distances between the densities are reduced to the differences between their covariance matrices (Table 5). With the 2Wasserstein distance, the distances between the densities are reduced to the Hilbert-Schmidt distances between the square root of the covariance matrices while the dual STATIS method (Lavit et al., 1994) uses Hilbert-Schmidt distances between the covariance matrices. In R, the calculations can partly be performed with the DSTATIS function of the multigroup package (Eslami et al., 2013, 2020) or the statis function of the ade4 package (Dray et al., 2007) after some transformation. However, the outputs of these two functions are completely different from those of the fmdsd function (MDS of densities Section): fmdsd focuses on the visualization of the occasions, while the other functions focus more on the visualization of variables or individuals.

data. scaled $=$ TRUE

With the two previous special cases, we have shown that MDS on densities is a way to take into account globally the means, variances, and covariances of the occasions and, therefore, a form of generalization of separate analyses either on averages or on variances and covariances. The optional argument data.scaled is useful when the analyst is interested to focus only on the relationships between variables.

\section{Appendix F: DA of densities and classic DA}

\section{Densities associated to the classes of the factor $G$ defined on the occasions}

In the functions fdiscd.misclass and fdiscd.predict, for each $k=1, \ldots, K$, the density $g_{k}$ representing the class $k$ of $G$ is estimated using a procedure selected by means of the argument crit. Recalling that the class $k$ contains $T_{k}$ densities $f_{t}$, the three procedures are defined as follows.

1. All samples related to the $T_{k}$ occasions of class $k$ are pooled and constitute a single sample, which is then used to estimate $g_{k}$.

2. If $\hat{f}_{t}$ estimates $f_{t}$, then $g_{k}$ is estimated by the mean value of the $T_{k}$ densities pertaining to the class $k:\left(1 / T_{k}\right) \sum \hat{f}_{t}$.

3. The mean value of the previous $T_{k}$ densities is calculated by weighting each $\hat{f}_{t}$ by the size of its corresponding sample: $\left(1 / \sum n_{t}\right) \sum n_{t} \hat{f}_{t}$.

The last two procedures are only available if the argument distance is set to "12". If there is only one occasion per class that is $T_{k}=1, \forall k$, the three procedures are the same. In this case, the data are those of the training step of classic DA. However, in the prediction step, we have to assign a group of individuals not individually but taken as a whole.

\section{Homoscedatic Gaussian case}

We assume that:

- the densities $f_{t}(t=1, \ldots, T)$ are Gaussian with the same covariance matrix $V$. We denote $f_{t}$ by $N\left(m_{t}, V\right)$;

- there is one density $f_{t}$ per class of $G$. So, there are $K=T$ classes, and the density of the class $k$ is denoted by $f_{k}$.

- the density $f_{T+1}$ is Gaussian $N\left(m_{T+1}, V\right)$

We have to predict the class value of $f_{T+1}$ using the rule 4 and one of the distances from Table 5. For distance $=$ "wasserstein", we calculate the distances between the mean vectors $\left\|m_{T+1}-m_{k}\right\|_{I_{p}}(k=$ 
$1, \ldots, T)$, and for the other distances we calculate the distances $\left\|m_{T+1}-m_{k}\right\|_{V^{-1}}(k=1, \ldots, T)$.

In the homoscedastic Gaussian case, this makes DA of densities appear as a form of extension of linear discriminant analysis where the group of individuals to be predicted is summarized by the mean vector of the group.

\section{Appendix G: Some computation times}

All the following calculations are carried out using a laptop computer equipped with an i5 processor on the archaeological data. The stone characteristics are stored in the data frame $x$. df. Then, we create the corresponding folder $\mathrm{x}$. folder.

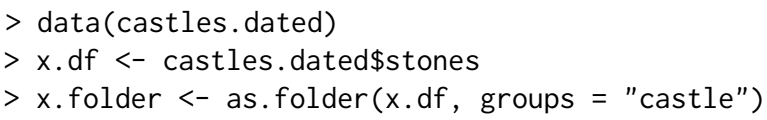

\section{Example 1: Comparison of distance indices in the continuous case}

The computation times of the inter-group distances are of the same magnitude when the densities are supposed Gaussian and parametrically estimated. Computations with the $L^{2}$ distance take about one second.

$>$ system.time(matdistl2d(x.folder, method = "gaussiand"))

$$
\begin{array}{rrr}
\text { user } & \text { system } & \text { elapsed } \\
1.13 & 0.06 & 1.36
\end{array}
$$

When the densities are estimated by the kernel method, the computation time is multiplied by about 5 .

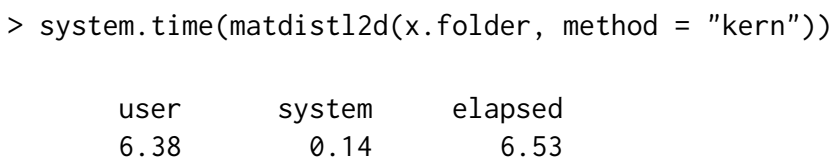

\section{Example 2: Computation time of the function fdiscd.misclass}

We build the hierarchical folder $x$. fh corresponding to the archeological data. We first apply the function fdiscd.misclass with the option gaussiand = TRUE then with the option gaussiand = FALSE.

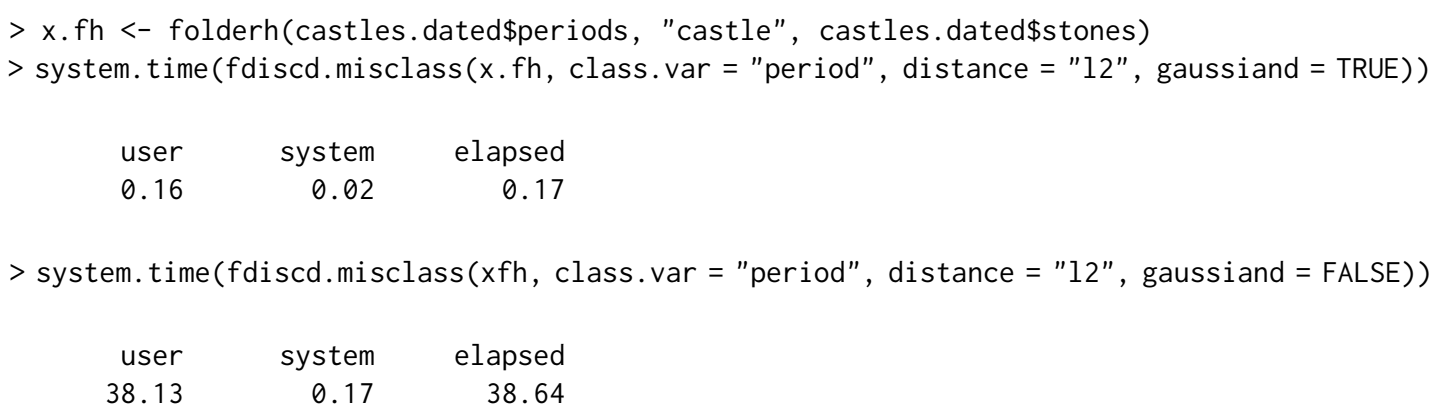

\section{Appendix H: MDS on compositional data and compositional data analysis}

The discrete densities considered in dad are compositional data as they are made up of positive numbers whose sums are one. It is possible to apply to them the techniques developed in the package compositions after an adapted data formatting work. The opposite, i.e., applying dad techniques to compositional data, is partly true as in the following example illustrating the functions princomp. acomp of compositions and mdsdd of dad on the same data. 

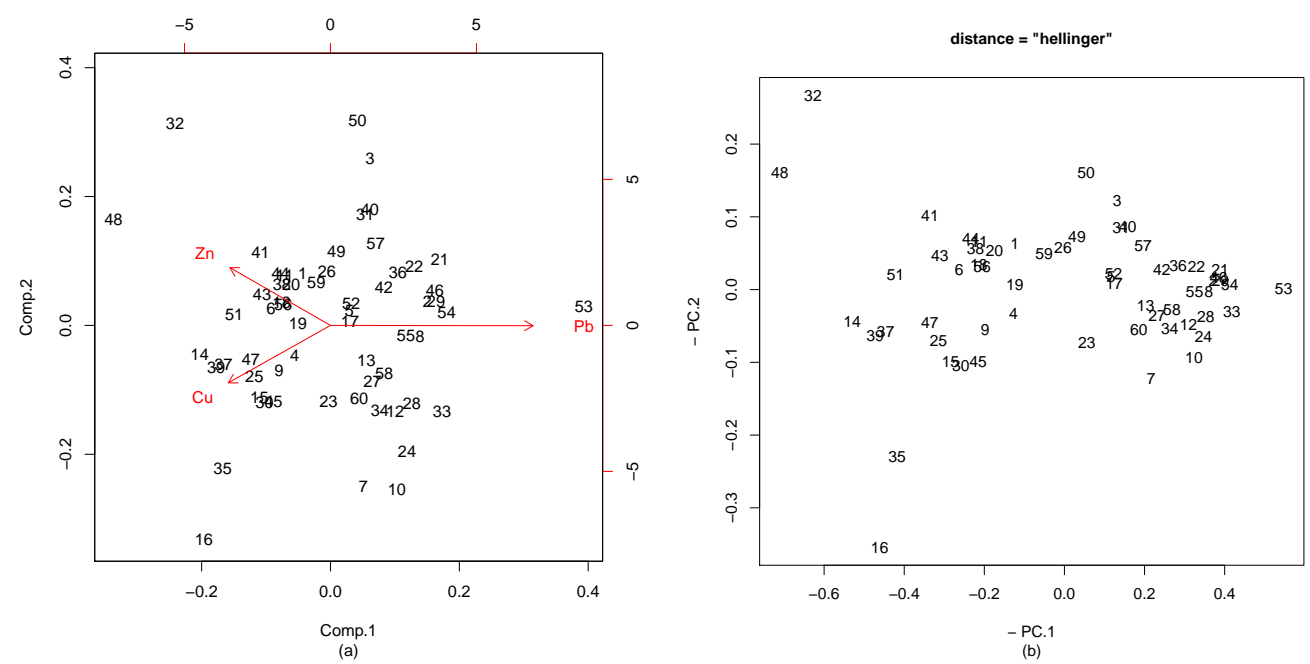

Figure 7: Compositional data. (a) The two first principal components of princomp. acomp applied on sa. lognormals data. (b) The two first principal coordinates of mdsdd on the same data. The signs of the scores are chosen so that the comparison of the two graphs is easier.
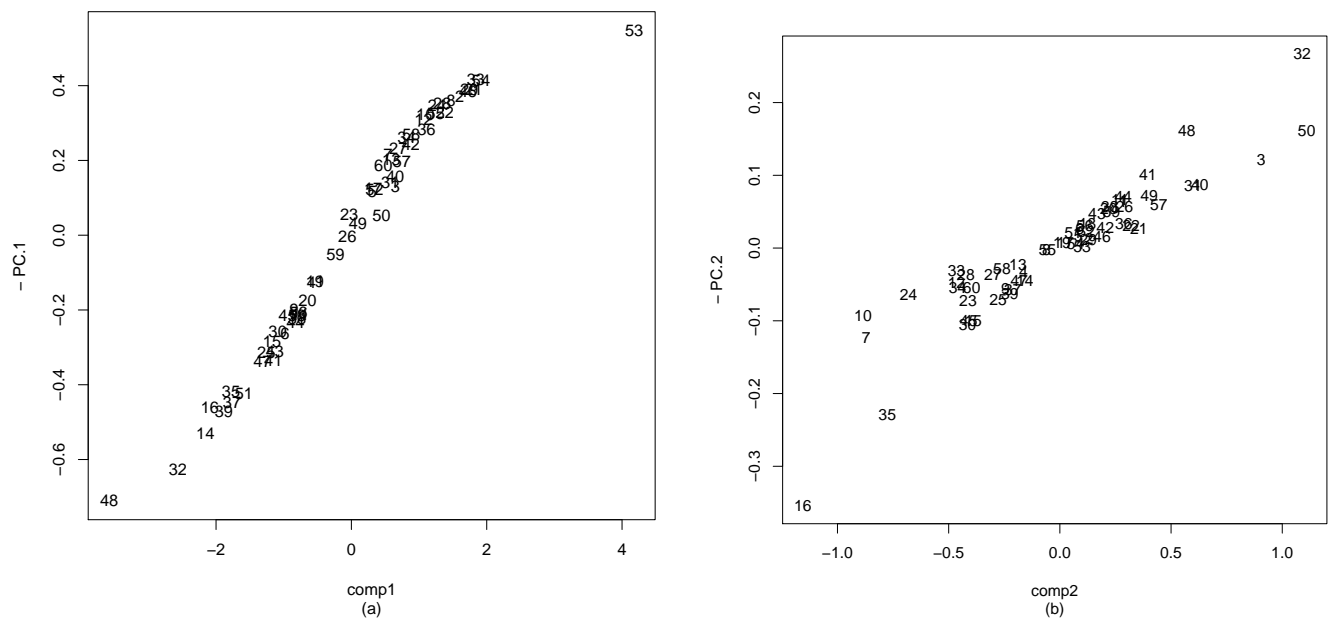

Figure 8: Plots of the scores of the figure $7 \mathrm{a}$ and those of the figure $7 \mathrm{~b}$. The correlation between comp1 and -PC. 1 is 0.98 and between comp2 and -PC. 2 is 0.93 .

\section{Data loading}

The simulated data sa. lognormals of compositions are stored in a matrix $60 \times 3$ whose columns are the amounts of $\mathrm{Cu}, \mathrm{Zn}$, and $\mathrm{Pb}$ present in 60 samples. They are loaded as follows.

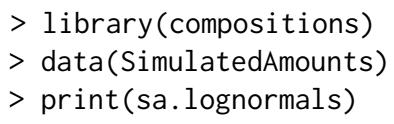

$\begin{array}{lrrr} & \mathrm{Cu} & \mathrm{Zn} & \mathrm{Pb} \\ {[1,]} & 8.8043262 & 35.1671810 & 45.895025 \\ \ldots & & & \\ {[60,]} & 3.9854998 & 6.1301909 & 40.579417\end{array}$

These initial data are transformed so that each row is of sum 1 by means of the acomp function.

$>\operatorname{acomp}(\mathrm{sa} \cdot \operatorname{lognormals}) \rightarrow \mathrm{x} 1$
$>\operatorname{print}(\mathrm{x} 1)$ 


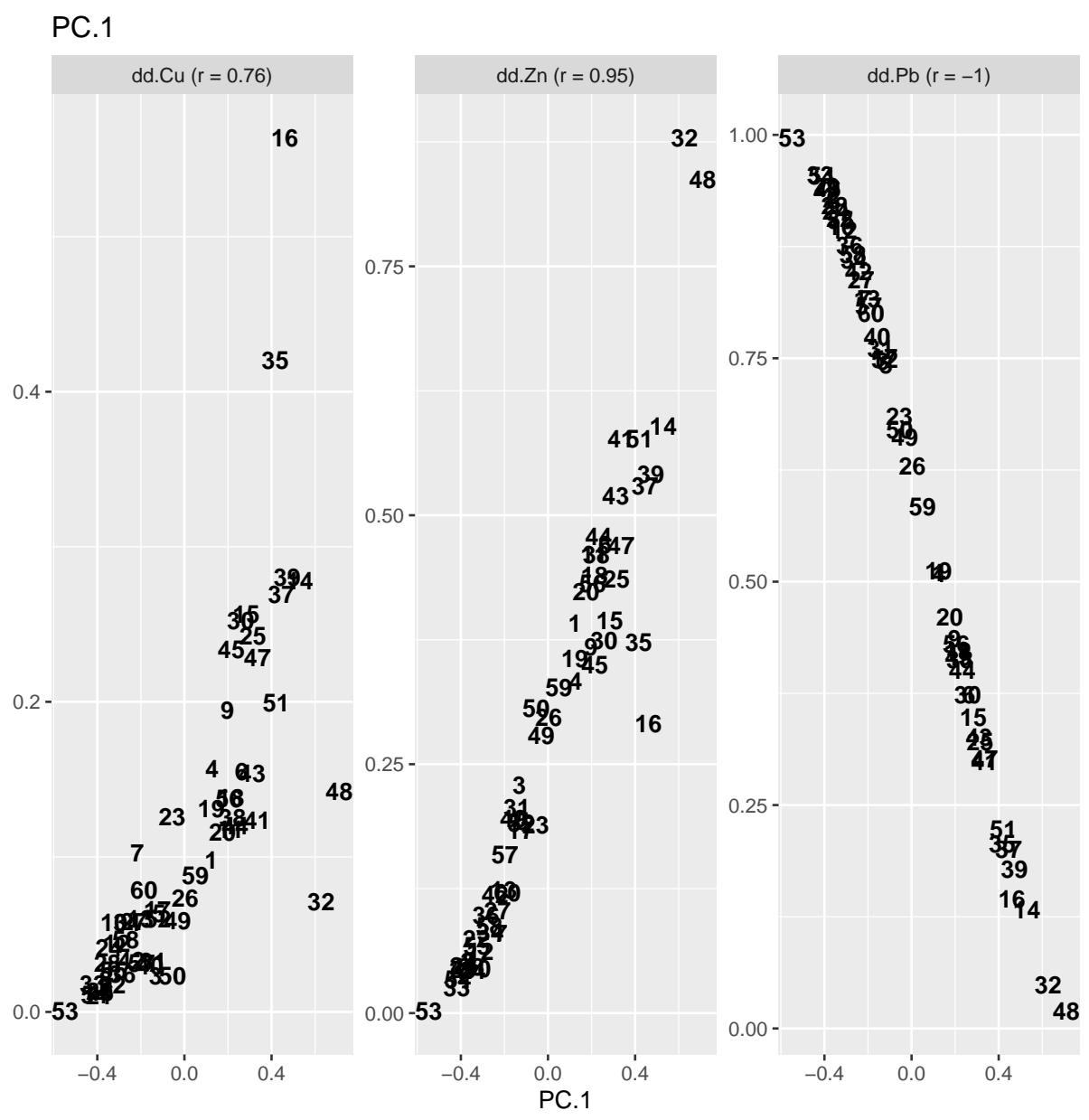

Figure 9: MDS of compositional data considered as discrete densities. Plotting PC. 1 against the relative amounts of $\mathrm{Cu}, \mathrm{Zn}$, and $\mathrm{Pb}$. 
$\mathrm{Cu} \quad \mathrm{Zn} \quad \mathrm{Pb}$

$[1] 0.097971136 \quad$,

$\ldots$

$[60] \quad$,

$\operatorname{attr}($, "class")

[1] acomp

The rows of the object $\mathrm{x} 1$ of class acomp (relative amounts of $\mathrm{Cu}, \mathrm{Zn}$, and Pb present in 60 samples) are transformed into tables or arrays. These tables or arrays then are organized in a list to be subjected to mdsdd. The three levels of the unique categorical variable are denoted dd. $\mathrm{Cu}, \mathrm{dd} . \mathrm{Zn}$, and dd. Pb.

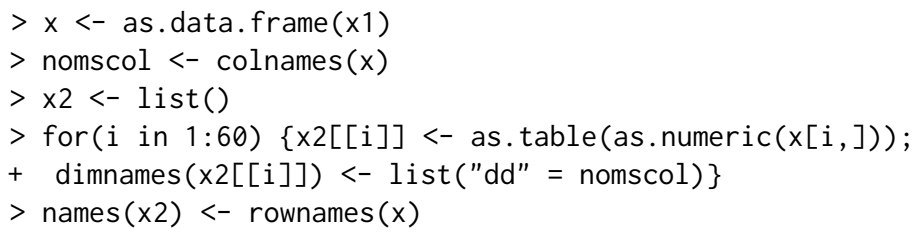

\section{Outputs of princomp and mdsdd}

By applying the princomp. acomp (or simply princomp) function to $\mathrm{x} 1$, we obtain the visualization of the data in a biplot (Fig. 7a).

The mdsdd function (MDS of discrete densities) is applied to $\times 2$ which are the rows of $x 1$ considered as discrete densities. We choose to present the results for the distance argument set to hellinger. These results are quite similar to those obtained with other distances as chisqsym, jeffreys, or jensen.

$r 2=\operatorname{mdsdd}(x 2$, distance $=$ "hellinger")

It provides the figure $7 \mathrm{~b}$.

The two figures are almost equivalent: by plotting the coordinates comp1 and comp2 against PC. 1 and PC. 2 of the two previous graphics we get the figure 8 .

\section{Interpretation of the scores}

For the interpretation of the axes, the two packages provide quite different tools. With compositions, the biplot makes it possible to visualize the links between the variables $\mathrm{Cu}, \mathrm{Zn}$, and $\mathrm{Pb}$ and their links with the principal components. With dad, the interpretation is done by crossing the scores and the initial data. The interpretation is based on the strength of the links between the PCs and the probabilities of occurence of each level. In the compositional data example, almost a single axis would suffice to explain the general trend: the first principal coordinate PC. 1 explains $90 \%$ of the inertia.

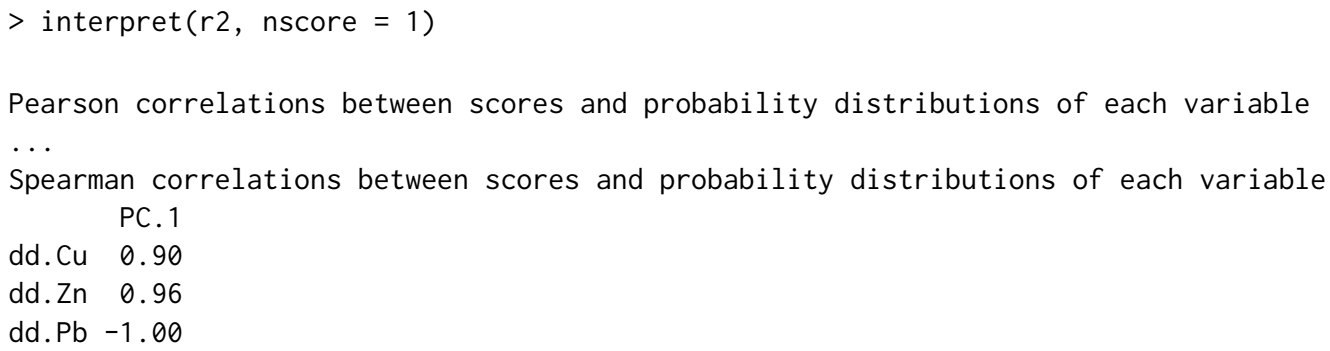

The graphical output is shown in Figure 9. The relative amounts are highly correlated with PC.1, showing that a low relative amount of the dd. $\mathrm{Pb}$ level corresponds to a high relative amount of the levels dd. Cu or dd. Zn. It is the result suggested by the biplot (Fig. 7a) returned by the function princomp. acomp of the package compositions.

\section{Bibliography}

J. Aitchison. The Statistical Analysis of Compositional Data. Chapman \& Hall, London, 1986. URL http://links.jstor.org/sici?sici=0035-9246\%281982\%2944\%3A2\%3C139\%3ATSAOCD\%3E2.0. CO\%3B2-9. [p194] 
R. Boumaza. Analyse en composantes principales de distributions gaussiennes multidimensionnelles. Revue de Statistique Appliquée, XLVI(2):5-20, 1998. URL http: / / www . numdam . org/i tem? id=RSA_1998_ _46_2_5_0. [p186]

R. Boumaza. Discriminant analysis with independently repeated multivariate measurements: An $l^{2}$ approach. Computational Statistics and Data Analysis, 47(4):823-843, 2004. URL https: //doi . org/10. 1016/j.csda.2004.01.001. [p179, 191]

R. Boumaza, S. Yousfi, and S. Demotes-Mainard. Interpreting the principal component analysis of multivariate density functions. Communications in Statistics - Theory and Methods, 44(16):3321-3339, 2015. URL https://doi.org/10.1080/03610926.2013.824103. [p186]

R. Boumaza, P. Santagostini, S. Yousfi, G. Hunault, J. Bourbeillon, B. Pumo, and S. Demotes-Mainard. dad: Three-Way / Multigroup Data Analysis Through Densities, 2021. URL https: //CRAN. R-project. $\mathrm{org} /$ package=dad. R Package version 3.4.6. [p179]

T. Cox and M. Cox. Multidimensional scaling. Chapman \& Hall/CRC, Boca Raton, 2001. URL https: //www. bibsonomy.org/bibtex/29a4eb97dc9592c07e19b9c4d8828a91a. [p186]

P. Delicado. Dimensionality reduction when data are density functions. Computational Statistics and Data Analysis, 55:401-420, 2011. URL https://doi .org/10.1016/j.csda.2010.05.008. [p186, 195]

M. Deza and E. Deza. Encyclopedia of Distances. Springer-Verlag, Heidelberg, 2013. URL https: //doi.org/10.1007/978-3-642-00234-2_1. [p179, 183]

S. Dray, A.-B. Dufour, and D. Chessel. The ade4 package - II: Two-table and K-table methods. R News, 7(2):47-52, 2007. URL https://cran.r-project.org/doc/Rnews/. [p179, 194, 201]

A. Eslami, E. M. Qannari, A. Kohler, and S. Bougeard. General overview of methods of analysis of multi-group datasets. Revue des Nouvelles Technologies de l'Information, 25:108-123, 2013. URL https://editions-rnti.fr/?inprocid=1001883. [p201]

A. Eslami, E. M. Qannari, S. Bougeard, and G. Sanchez. multigroup: Multigroup Data Analysis, 2020. URL https: //CRAN.R-project.org/package=multigroup. R package version 0.4.5. [p201]

M. Febrero-Bande and M. O. de la Fuente. Statistical computing in functional data analysis: The r package fda.usc. Journal of Statistical Software, 51(4):1-28, 2012. URL http: //www. jstatsoft. org/ v51/i04/. [p179]

P. Filzmoser, K. Hron, and M. Templ. Applied Compositional Data Analysis. With Worked Examples in R. Springer International Publishing. Springer Nature Switzerland AG, Cham, Switzerland, 2018. ISBN 978-3-319-96420-1. Springer Series in Statistics. [p179]

G. Gan, C. Ma, and J. Wu. Data Clustering: Theory, Algorithms and Applications. ASA-SIAM Series on Statistics and Applied Probability, SIAM,Philadelphia, ASA, Alexandria, VA, 2007. ISBN 0898716233. [p190]

C. Godin and Y. Caraglio. A multiscale model of plant topological structures. Journal of Theoretical Biology, 191(1):1-46, 1998. URL https://doi.org/10.1006/jtbi.1997.0561. [p199]

INSEE. Population active de 25 à 54 ans ayant un emploi et chômeurs par catégorie socioprofessionnelle et diplôme par commune et département (1968 à 2015), 2018. URL http: //www. insee. fr/fr/ statistiques $/ 1893185$. Accessed $=2019-06-25$. [p182]

H. Kiers. Towards a standardized notation and terminology in multiway analysis. Journal of Chemometrics, 14:105-122, 2000. URL https://doi .org/10.1002/1099-128X(200005/06)14:3<105: : AIDCEM582>3.0.C0;2-I. [p199]

A. Kneip and K. Utikal. Inference for density families using functional principal component analysis. Journal of the American Statistical Association, 96:519-542, 2001. URL https://doi .org/10.1198/ 016214501753168235. [p186]

W. Krzanowski. Principles of Multivariate Analysis. Oxford University Press, New-York, 1988. ISBN 978-0198507086. [p179]

C. Lavit, Y. Escoufier, R. Sabatier, and P. Traissac. The act (statis method). Computational Statistics $\mathcal{E}$ Data Analysis, 18:97-119, 1994. URL https://doi .org/10.1016/0167-9473(94)90134-1. [p201]

S. Lê, J. Josse, and F. Husson. FactoMineR: An r package for multivariate analysis. Journal of Statistical Software, 25(1):1-18, 2008. URL https://doi.org/10.18637/jss.v025.i01. [p179, 194] 
M. Maechler, P. Rousseeuw, A. Struyf, M. Hubert, and K. Hornik. cluster: Cluster Analysis Basics and Extensions, 2019. URL https: //CRAN. R-project. org/package=cluster. R package version 2.1.0 For new features, see the 'Changelog' file (in the package source). [p179, 194]

K. Mardia, J. Kent, and J. Bibby. Multivariate Analysis. Academic Press, London, 1979. ISBN 0-12471252-5. [p179]

A. Petersen, P. Z. Hadjipantelis, and H. G. Mueller. fdadensity: Functional Data Analysis for Density Functions by Transformation to a Hilbert Space, 2019. URL https://CRAN. R-project.org/package= fdadensity. R package version 0.1.2. [p179]

C. Pradal and T. Cokelaer. VPlants Documentation. Release 0.8. INRIA, France, 2010. URL http: //openalea.gforge.inria.fr/doc/vplants/newmtg/doc/_build/html/contents.html. [p199]

J. O. Ramsay, S. Graves, and G. Hooker. fda: Functional Data Analysis, 2020. URL https: //CRAN. Rproject. org/package=fda. R Package version 5.1.4. [p179]

J. Rudrauf and R. Boumaza. Contribution à l'étude de l'architecture médiévale: les caractéristiques des pierres à bossage des châteaux forts alsaciens. Centre de Recherches Archéologiques Médiévales de Saverne, 5:5-38, 2001. URL http://www. crams.fr/publications_detail.php?parPUBL=CFA05\# ancDebut. [p181, 192, 197]

M. Tsagris and G. Athineou. Compositional: Compositional Data Analysis, 2020. URL https://CRAN. Rproject. org/package=Compositional. R package version 3.8. [p179]

K. G. van den Boogaart, R. Tolosana-Delgado, and M. Bren. compositions: Compositional Data Analysis, 2020. URL https: //CRAN. R-project.org/package=compositions. R package version 1.40-5. [p179]

W. Venables and B. Ripley. Modern Applied Statistics with S. Springer-Verlag, New York, fourth edition, 2002. URL http://www. stats.ox.ac.uk/pub/MASS4. ISBN 0-387-95457-0. [p179, 194]

M. Wand and M. Jones. Kernel Smoothing. Chapman and Hall, London, 1995. ISBN 9780412552700. [p185]

S. Yousfi, R. Boumaza, D. Aissani, and S. Adjabi. Optimal bandwith matrices in functional principal component analysis of density functions. Journal of Statistical Computational and Simulation, 85(11): 2315-2330, 2015. URL https://doi.org/10.1080/00949655.2014.928293. [p186, 194]

Rachid BOUMAZA

IRHS-UMR1345, Université d'Angers, INRAE, Institut Agro, SFR 4207 QuaSaV

49071, Beaucouzé

France

ORCiD: 0000-0002-1600-1820

rachid.boumaza@agrocampus-ouest.fr

Pierre SANTAGOSTINI

IRHS-UMR1345, Université d'Angers, INRAE, Institut Agro, SFR 4207 QuaSaV 49071, Beaucouzé

France

pierre.santagostini@agrocampus-ouest.fr

Smail YOUSFI

Département de mathématiques

Université Mouloud Mammeri

Tizi-Ouzou

Algeria

smail_yousfi@ymail.com

Sabine DEMOTES-MAINARD

IRHS-UMR1345, Université d'Angers, INRAE, Institut Agro, SFR 4207 QuaSaV

49071, Beaucouzé

France

sabine. demotes-mainard@inrae. fr 